\title{
Adiabatically Correcting an Eddy-Permitting Model Using Large-Scale Hydrographic Data: Application to the Gulf Stream and the North Atlantic Current
}

\author{
Carsten Eden* and Richard J. Greatbatch \\ Department of Oceanography, Dalhousie University, Halifax, Nova Scotia, Canada \\ Claus W. Böning \\ Institut für Meereskunde, Kiel, Germany
}

(Manuscript received 18 September 2002, in final form 22 August 2003)

\begin{abstract}
This study focuses on an important aspect of air-sea interaction in models, namely, large-scale, spurious heat fluxes due to false pathways of the Gulf Stream and North Atlantic Current (NAC) in the "storm formation region" south and east of Newfoundland. Although high-resolution eddy-resolving models show some improvement in this respect, results are sensitive to poorly understood, subgrid-scale processes for which there is currently no complete, physically based parameterization. A simple method to correct an ocean general circulation model (OGCM), acting as a practical substitute for a physically based parameterization, is explored: the recently proposed "semiprognostic method," a technique for adiabatically adjusting flow properties of a hydrostatic OGCM. The authors show that application of the method to an eddy-permitting model of the North Atlantic Ocean yields more realistic flow patterns and watermass characteristics in the Gulf Stream and NAC regions; in particular, spurious surface heat fluxes are reduced. Four simple modifications to the method are proposed, and their benefits are demonstrated. The modifications successfully account for three drawbacks of the original method: reduced geostrophic wave speeds, damped mesoscale eddy activity, and spurious interaction with topography. It is argued that use of a corrected (eddy permitting) OGCM in a coupled modeling system for simulating present climate (as now becomes possible because of increasing computer power) should lead to a more realistic simulation in regions of strong air-sea interaction as compared with that obtained with an uncorrected model. The method is also well suited for the simulation of the uptake and transport of passive tracers, such as anthropogenic carbon dioxide or components of ecosystem models.
\end{abstract}

\section{Introduction}

Any "useful" model of the ocean has to exclude processes, by either simply neglecting or parameterizing them, and therefore contains systematic errors. In a "good" ocean model, however, effects of these errors should be "small." In this study, the process under focus is the large-scale, time-mean circulation of the North Atlantic Ocean and the model under consideration is a standard, numerical, eddy-permitting ocean general circulation model (OGCM). We have in mind, as a possible application of the OGCM, a climate prediction framework in which the ocean model is coupled to other components of the climate system, for example, the atmosphere and cryosphere. For decadal-scale integra-

* Current affiliation: Institut für Meereskunde, Kiel, Germany.

Corresponding author address: Carsten Eden, Institut für Meereskunde, FB I, Theorie und Modellierung, Düsternbrooker Weg 20, 24105 Kiel, Germany.

E-mail: ceden@ifm.uni-kiel.de tions, present computational resources would allow the use of horizontal resolutions in the ocean model to nearly resolve the scales $(\sim 30 \mathrm{~km})$ of the vigorous mesoscale motions in the ocean (eddy-permitting models). However, we are concerned that the ocean model under consideration might contain systematic errors due to effects of unresolved processes on the large-scale circulation that are not small.

In an eddy-permitting model of the North Atlantic, one readily identifies spurious surface heat fluxes in the Gulf Stream region and in the path of the North Atlantic Current (NAC) around Newfoundland as the most severe bias of the model. Although eddy-resolving models show some improvements in this respect, the results still heavily depend on model details such as parameterizations for friction and diffusion or discretization of topography (e.g., Smith et al. 2000; Chassignet and Garraffo 2001), pointing toward processes involving still smaller horizontal scales, vertical scales, or topography, which are not yet understood. The most sensitive points of the flow pattern appear to be the Gulf Stream separation and the attachment of the NAC to the topo- 
graphic slope southeast of Newfoundland. However, we want to stress that this region (sometimes referred to as the storm formation region) is of potential importance for a coupled model since, here, strong baroclinicity in the lower atmosphere is maintained by the flux of sensible and latent heat out of the ocean, supporting growing disturbances and influencing the North Atlantic storm strack (Hoskins and Valdes 1990).

The systematic deficiency in the western boundary flow pattern of even "realistic," high-resolution OGCMs clearly demands concerted research efforts toward an improved understanding of the underlying processes and a physically more realistic representation or parameterization in (coupled) models. However, in the absence of a proper understanding and parameterization of subgrid-scale processes in the western boundary region, we also need ways to reduce model errors associated with these processes. In this study we explore a simple method to correct an OGCM for systematic errors. In effect, we are suggesting a practical substitute for a physically based parameterization. The method we use is based on the "semiprognostic" method proposed by Sheng et al. (2001): a simple way to adiabatically change the advection properties of a hydrostatic OGCM by altering the pressure gradient seen by the model in the momentum balance. The method can be viewed as a simple technique to assimilate hydrographic data into an ocean model, with the advantage over other simple methods, for example, the robust diagnostic method of Sarmiento and Bryan (1982), that no spurious diabatic sources and sinks are introduced.

Sheng et al. (2001) applied the method with good success to a regional model of the northwestern Atlantic. Here, we extend the approach to a basin-scale application. Although the basic method proposed by Sheng et al. performs well in improving the simulation of circulation patterns and watermass characteristics in the North Atlantic in comparison with observations, some problems arise in the model. Among them are changed dynamical properties of the semiprognostic OGCM, for instance, reduced Rossby wave speeds and damped eddy activity. We propose four modifications of the basic scheme to overcome these difficulties and to demonstrate the benefits of the modifications in the model.

As an end product, we obtain an ocean model containing a fixed, non-flow-interactive correction term in the momentum balance, taking the place of a parameterization of effects of unresolved processes. The corrected model yields improved simulations of flow structures and watermass characteristics, especially in the northwestern North Atlantic (the storm formation region) with the result that the corrected OGCM is better suited to be used in a coupled model system of the present climate than the uncorrected one. We note, however, that the use of the method is restricted to small deviations from the present climate because of the use of present-day observational data. Since the (adiabatic) correction does not affect the tracer budgets directly, it is also well suited for a more realistic simulation of the uptake and transport of passive tracers, such as, for example, anthropogenic carbon dioxide $\left(\mathrm{CO}_{2}\right)$ or components of a pelagic ecosystem model.

This paper is structured as follows. In the second section we present the OGCM and review the semiprognostic method, followed by a discussion of four modifications to the method. In the third section, we describe results from the prognostic OGCM in comparison with other eddy-permitting and eddy-resolving models and discuss the application of the original semiprognostic and the modified versions. The last section discusses our conclusions.

\section{Ocean models and methods}

\section{a. Prognostic models}

We apply the semiprognostic method to an OGCM of the North Atlantic, part of the Family of Linked Atlantic Model Experiments (FLAME) hierarchy of models (Dengg et al. 1999), which includes versions of different resolution and different parameterizations of subgrid-scale processes. In all cases, however, the numerical $\operatorname{code}^{1}$ is based on a revised version of Geophysical Fluid Dynamics Laboratory (GFDL) Modular Ocean Model 2 (MOM2; Pacanowski 1995). The present study focus on a FLAME configuration, which we call the "eddy-permitting (FLAME) model", spanning the Atlantic Ocean from $20^{\circ} \mathrm{S}$ to $70^{\circ} \mathrm{N}$ with a horizontal resolution of $1 / 3^{\circ} \cos \phi$ ( $\phi$ denoting latitude). The configuration is almost identical to the $z$-level model that was part of the European Dynamics of North Atlantic Models (DYNAMO) ocean model intercomparison project (Willebrand et al. 2001). In particular, it uses the same horizontal resolution (eddy permitting), the same surface boundary forcing [Haney-type heat flux condition as given by Barnier et al. (1995) and a restoring condition for sea surface salinity], and the same lateral boundary conditions [open boundaries after Stevens (1990) along $20^{\circ} \mathrm{S}$ and a buoyancy-restoring zone north of the Greenland-Iceland-Scotland ridge system and in the Gulf of Cadiz] as in DYNAMO. The main differences in the present FLAME setup are increased vertical resolution (45 levels) and therefore newly interpolated topography. In this study, we also change some of the physical parameterizations and numerical schemes. A third-order tracer advection scheme (Quicker) replaces the traditional second-order scheme [see Griffies et al. (2000) for the benefits] and a closure for the vertical turbulent kinetic energy following Gaspar et al. (1990) [utilizing identical parameters for the scheme as in Oschlies and Garcon (1999); see also a description of the model improvement therein] replaces a scheme pro-

\footnotetext{
${ }^{1}$ The numerical code together with all configurations used in this study can be accessed online at http://www.ifm.uni-kiel.de/fb/fb1/tm/ data/pers/ceden/spflame/index.html.
} 


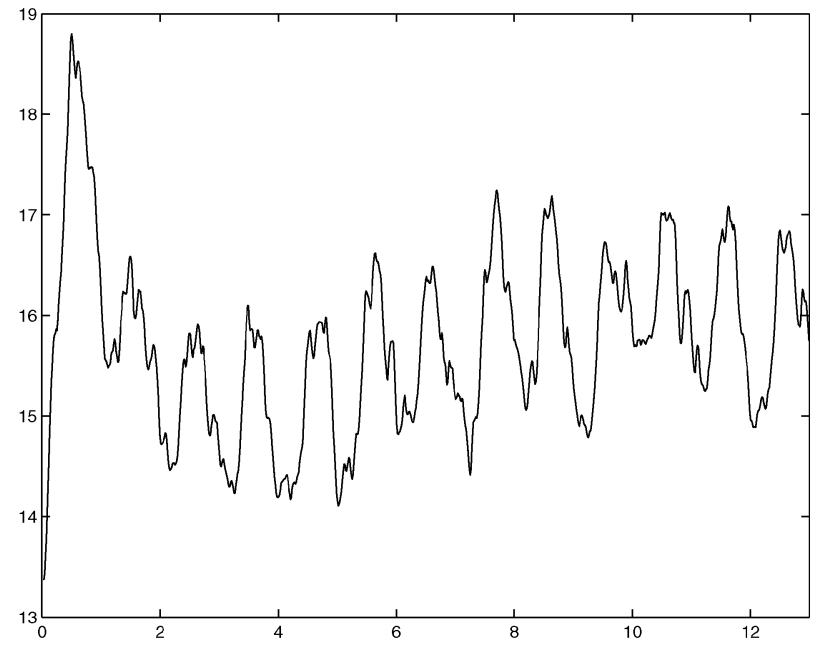

FIG. 1. Basin-averaged kinetic energy density $\left(\mathrm{cm}^{2} \mathrm{~s}^{2}\right)$ over the reference integration (13 yr) of the eddy-permitting prognostic model.

posed by Gargett (1984). Effects of unresolved processes in the momentum balance are parameterized, as in the $z$-level DYNAMO model, using biharmonic friction with viscosity of $2.5 \times 10^{11} \cos \phi \mathrm{m}^{4} \mathrm{~s}^{-1}$. Explicit lateral diffusion is, in contrast to DYNAMO, set to zero; bottom friction is the same as in DYNAMO.

In each experiment the eddy-permitting FLAME model is integrated for a $10-y r$ spinup period before being analyzed, unless otherwise noted. Annual mean model fields are obtained by averaging over a subsequent 3-yr integration. The spinup period may appear short, if one considers that the bulk of the baroclinic adjustment of a North Atlantic model is believed to take place in 10-15 years. However, to explore the semiprognostic method, we have decided to invest our restricted resources in several, shorter experiments with the eddypermitting OGCM, instead of only a few, but longer experiments. For clarification, Fig. 1 shows the basinaveraged kinetic energy in a 13-yr-long integration of the prognostic eddy-permitting FLAME model, giving an indication of the dynamical adjustment time scale in the model. Note that the time series in Fig. 1 saturates after about 10 years of integration and shows no large trend for the 3-yr analysis period.

For comparison we use results of the z-level DYNAMO model and an eddy-resolving FLAME model version. The latter model uses the same domain, same surface forcing and lateral boundary conditions, and the same vertical resolution as the eddy-permitting FLAME model but adopts a drastically increased horizontal resolution $\left(1 / 12^{\circ} \cos \phi\right)$. Setup and spinup procedures of the eddy-resolving model are discussed in detail in Eden and Böning (2002); here we want to note the following caveat. The eddy-resolving FLAME model was initialized with the state of the eddy-permitting version at the end of a 15-yr integration. The eddy-resolving version was then integrated for 8 years, subdivided in two pe- riods, 3 years with high and 5 years with low viscosity and diffusivity. Results are shown here as 3-yr averages from the end of the latter period. This integration may appear to be too short for a basin-scale baroclinic adjustment, but we think, nevertheless, that comparison aids the discussion in this study to show some effects of increased horizontal resolution since it utilizes an identical configuration as the eddy-permitting model.

\section{b. Original semiprognostic method}

Before describing the semiprognostic method in detail, we want to motivate its name here in passing. A "prognostic" ocean model predicts momentum as well as the dynamical active density. In contrast, a "diagnostic" model is an OGCM in which potential temperature and salinity (density) are held fixed at certain climatological values. The momentum remains then as the only prognostic variable. There are certain disadvantages involved with such an approach, as discussed by, for example, Greatbatch et al. (1991) and Ezer and Mellor (1994). Because of small discrepancies between the prescribed baroclinic structure and the discretized model topography, large spurious currents can occur since the density structure cannot be adjusted as in a freely evolving prognostic model. In an attempt to overcome this problem, model density is sometimes relaxed toward a climatology on a short time scale of order of days ("nudging"), which is then called a "robust diagnostic" model (Sarmiento and Bryan 1982). However, it is obvious that unphysical sources and sinks of heat and salt are introduced by such an approach (Marotzke and Willebrand 1996).

In a "semiprognostic" model ${ }^{2}$ (Sheng et al. 2001) the dynamical active density is given as a linear combination of an a priori known (in situ) density $\left(\rho_{c}\right)$ and the density given by temperature and salinity (and pressure) calculated by the model using the equation of state $\left(\rho_{m}\right)$ :

$$
\rho^{*}=\alpha \rho_{m}+(1-\alpha) \rho_{c} .
$$

It is the linear combination $\rho^{*}$ that is used in the hydrostatic equation of a semiprognostic model to calculate the pressure force in the baroclinic momentum equation. This is the only difference from a conventional, prognostic OGCM and is obviously readily implemented in numerical code. The parameter $\alpha$ ranges between 0 and 1. For $\alpha=1$ we recover the prognostic model, for $\alpha=0$ we get a diagnostic model, and for values of the parameter between 0 and 1 we get a semiprognostic model.

Sheng et al. (2001) use monthly mean climatological values of temperature and salinity to calculate the a priori known density $\rho_{c}$. They use the best linear unbiased estimator to choose a value for $\alpha$ by comparing

\footnotetext{
${ }^{2}$ For being in between a diagnostic and prognostic model. One could also name it "semidiagnostic." We prefer the optimistic way.
} 
velocities from the pure diagnostic and prognostic model runs with observations. On average, they find $\alpha=0.5$ to be the most appropriate value. An alternative approach would be to minimize a cost function using tracer observations and an adjoint version of the semiprognostic model. However, this approach is beyond the scope of the present study and is left for future research. In the present study, we shall take $\alpha=0.5$ throughout the experiments and also use monthly mean climatological temperature and salinity to compute $\rho_{c}$. However, in contrast to Sheng et al. (2001), who use a climatology of the northwest Atlantic (Geshelin et al. 1999), we use a combination of the global climatologies given by Boyer and Levitus (1997) and Levitus and Boyer (1994), serving also as the initial condition for the model. The seasonal cycle contained in the monthly mean $1^{\circ} \times 1^{\circ}$ climatology of Levitus and Boyer (1994) was extracted and carefully applied to the annual mean $1 / 4^{\circ} \times 1 / 4^{\circ}$ climatology of Boyer and Levitus (1997), in order to obtain both high temporal and high spatial resolution. Monthly mean temperature and salinity are linearly interpolated to the model time step. More details of the procedure are given in FLAME Group (1998). ${ }^{3}$ Results from the semiprognostic model of Sheng et al. (2001) (which is similar to our OGCM in the present study, but restricted to the northwest Atlantic) are in several ways more realistic than results from the prognostic version of their OGCM. In particular, the circulation and watermass characteristics of the North Atlantic Current (NAC) east of the Grand Banks fit better to observations in the semiprognostic version. The less realistic simulation of this region in the prognostic version of the model is common to similar models of the North Atlantic and is a well-known deficiency of $z$-level models (Willebrand et al. 2001). We show in section 3 that the same deficiency occurs in our prognostic OGCM and is improved in the semiprognostic version of the model.

We want to stress that the success of the method, especially in improving the watermass characteristics, is by no means trivial since the tracer equations are unchanged in the semiprognostic model; that is, no artificial sources or sinks of tracers are introduced (nudging; Sarmiento and Bryan 1982). Note that the method is also different from "momentum nudging," as used by, for example, Woodgate and Killworth (1997) and Stutzer and Krauss (1998). In the semiprognostic method there are no artificial Newtonian relaxation terms added to the primitive equations. On the other hand, and for the same reason, the success of the method, or the convergence to a more realistic model state, is not guaranteed. Differences between model density $\rho_{m}$ and climatological density $\rho_{c}$ are driving changes in the advective flow due to changes in the

\footnotetext{
${ }^{3}$ This report can be accessed at ftp://ftp.ifm.uni-kiel.de/pub/ FLAME/WebDownload/Reports/FLAME_Rep98.ps.gz.
}

momentum balance. This changed flow may not lead to a smaller difference between $\rho_{m}$ and $\rho_{c}$, that is, a more realistic $\rho_{m}$. However, we have never encountered a diverging solution in a semiprognostic model; in all of our applications the method converges to a more realistic mean model state and appears surprisingly robust. This holds also for the modified versions of the method, which we discuss next.

\section{c. Modifications to the semiprognostic method}

There are certain drawbacks of a semiprognostic model, which we want to address and resolve in this study with modified versions of the method.

1) The pressure forcing in the momentum balance in the semiprognostic model can be written as

$$
\begin{aligned}
\boldsymbol{\nabla} p & =g \boldsymbol{\nabla} \int_{z}^{0} d z\left[\alpha \rho_{m}+(1-\alpha) \rho_{c}\right] \\
& =\alpha \boldsymbol{\nabla} p_{m}+(1-\alpha) \boldsymbol{\nabla} p_{c},
\end{aligned}
$$

introducing the pressure variables $p_{m}=g \int_{z}^{0} d z \rho_{m}$ and $p_{c}=g \int_{z}^{0} d z \rho_{c}$ and neglecting the contribution from the surface pressure. Considering now linear waves [small perturbations of a mean state in balance with the "forcing" term $(1-\alpha) \boldsymbol{\nabla} p_{c}$ ] it becomes clear that waves must be affected by $\alpha$; that is, it is easy to show that baroclinic gravity wave speeds are reduced by a factor $\sqrt{\alpha}$. This means that, for instance, long (flat bottom) Rossby wave speeds are reduced by $\alpha$ and baroclinic Kelvin wave speeds by $\sqrt{\alpha}$.

2) For the same reason, anomalous geostrophic velocities, that is, geostrophic eddies, are reduced by a factor $\alpha$. It is reasonable to expect, therefore, a similar damping influence on eddy kinetic energy.

3 ) Especially in regions of strong boundary currents, interaction of the semiprognostic method with the model topography can produce spurious up- and downwelling, affecting large-scale flow properties. We will show and further explain this effect in section 3.

We want to note that drawbacks 1 and 2 can be utilized as an analysis tool. Eden and Greatbatch (2003) use the diagnosed, monthly mean model density after a spinup phase of a prognostic North Atlantic model as the prescribed density $\rho_{c}$ (instead of an observed, climatological density) in a semiprognostic version of the model. The effect is that the mean state of the semiprognostic model is unchanged (with respect to the prognostic version). But, since drawbacks 1 and 2 still hold, Eden and Greatbatch (2003) are able to quantify the role of geostrophic waves and anomalous advection, in their case for the ocean's response to changing surface forcing.

However, for the purpose of a realistic simulation of the ocean such effects appear undesirable. A straightforward way to overcome problems 1 and 2 is to di- 
agnose the correction in Eq. (1) and to apply a corresponding non-flow-interactive correction in a subsequent integration. Equation (1) can be written as

$$
\rho^{*}=\rho_{m}+(1-\alpha)\left(\rho_{c}-\rho_{m}\right) .
$$

We simply diagnose [by calculating over three years a monthly climatology of $\left.(1-\alpha)\left(\rho_{c}-\rho_{m}\right)\right]$ the second term on the rhs of Eq. (2) in a semiprognostic model experiment. In the subsequent integration, we add these averages to the model density $\rho_{m}$ in the hydrostatic equation of the model, which then corresponds to a nonflow-interactive forcing term in the momentum balance. This (monthly varying) forcing term will contain the corrections made to the model by the semiprognostic method, with the difference that the correction is not flow interactive anymore. We may argue that this correction accounts for errors of the prognostic model and call this kind of model a corrected-prognostic model. We demonstrate such an approach with our eddy-permitting OGCM in section 3. Note that a corrected-prognostic model is "fully prognostic" again, and the influence of the semiprognostic method on waves, anomalous advection, and eddy kinetic energy is absent. The only difference from the conventional prognostic model is that we have derived a correction term in the momentum balance that accounts for the systematic errors of the model. In effect, this correction behaves similarly to a parameterization, accounting for unresolved processes that would lead, without correction or parameterization, to systematic errors in the model.

However, to derive this correction terms, it would be of benefit to have a semiprognostic method, which relies only on the large-scale density structure, while the baroclinic mesoscale structures remain unaffected. This would reduce or eliminate the damping influence on the resolved eddy activity in the model. It is also likely that errors (e.g., measurement errors, geophysical noise by mesoscale eddies, or insufficient smoothing techniques in the data processing) in the climatology used for the prescribed density $\rho_{c}$ show up predominantly on smaller scales. For this reason it would be desirable, as well, to use only the large-scale features of the climatological density in the semiprognostic method.

We propose two different approaches to realize such a semiprognostic method. The first relies on spatial averaging, the second on temporal averaging. To formulate the first, we add to Eq. (1) a scale-selective operator $P$, acting on the difference between dynamical active density $\rho^{*}$ and model-calculated density $\rho_{m}$ :

$$
\rho^{*}=\rho_{m}+(1-\alpha)\left(\rho_{c}-\rho_{m}\right)+P\left(\rho^{*}-\rho_{m}\right) .
$$

Here $P$ is supposed to be very large on small scales and very small on large scales. For the large scales we recover the original semiprognostic method, that is, Eq. (1), and for the small scales we get $\rho^{*}-\rho_{m}=0$ and

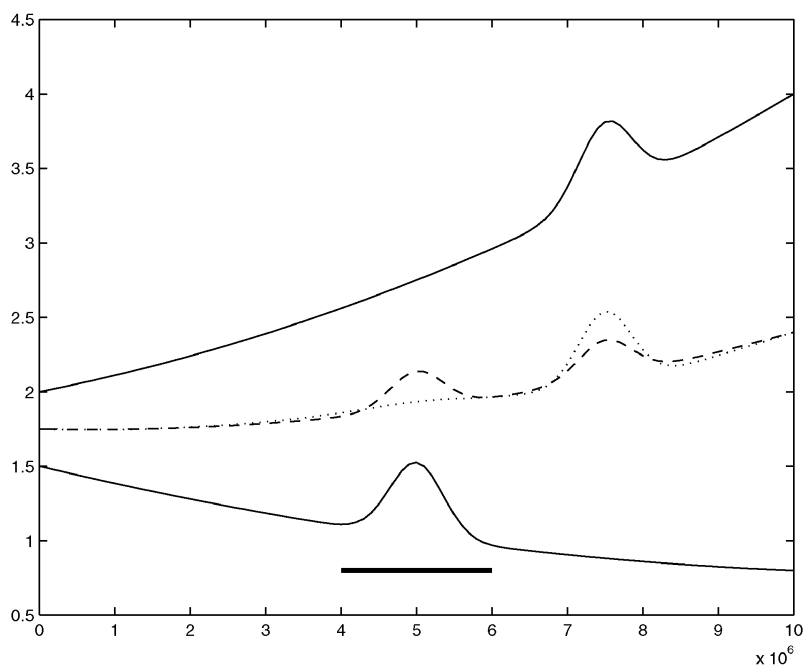

FIG. 2. A one-dimensional example illustrating the principal effect of the original and the smoothed semiprognostic methods on possible data errors and mesoscale (model) features. Shown are isopycnals of the model density $\rho_{m}$ (lower solid line), the climatological density $\rho_{c}$ (upper solid line), and the dynamically active density $\rho^{*}$ for the original semiprognostic method (dashed line) and the smoothed semiprognostic method (dotted line). Note that in contrast to the original method, the smoothed method preserves the idealized mesoscale feature in $\rho_{m}$ and suppresses the "data error" in $\rho_{c}$. Units for density (vertical axis) are arbitrary; the horizontal axis denotes a spatial dimension $(\mathrm{m})$.

thus no effect of the method ${ }^{4} ; P=L^{2} \nabla^{2}$ (or $P=L^{4} \nabla^{4}$ ) satisfies this condition, where $\nabla$ denotes the two-dimensional, horizontal nabla operator and $L$ is a length scale, separating between the damping and the negligible influence of $P$.

To illustrate the benefit of Eq. (3) we show a simple one-dimensional example in Fig. 2. The lower solid line in the figure denotes the model density $\rho_{m}$; the upper solid line the climatological density $\rho_{c}$. There are smallscale features in both densities, which are meant to resemble either a mesoscale eddy (for $\rho_{m}$ ) or a data error (for $\rho_{c}$ ). The dashed line in the middle shows the active density $\rho^{*}$ using Eq. (1) with $\alpha=0.5$. Both bumps, which we have built into the densities, show up in $\rho^{*}$, which then contains the "eddy" and the "data error." However, note that the amplitude of the eddy in $\rho_{m}$ is reduced by half. Using Eq. (3) to calculate $\rho^{*}$ (with $P$ $=L^{2} \nabla^{2}$, the thick solid line in the figure denotes the length scale $L$ ) yields the dotted line, in which the data error in $\rho_{c}$ is damped away and the eddy from $\rho_{m}$ is more or less preserved, while the large-scale gradient of $\rho^{*}$ still remains the same.

For the practical implementation of Eq. (3), it is necessary to solve as many Helmholtz equations as there

\footnotetext{
${ }^{4}$ Note that it is also possible to construct a method that acts predominantly on small scales while being negligible on large scales. Such an approach would be a simple method for, e.g., assimilating mesoscale signals into an (eddy permitting) ocean model. However, we do not explore this route in the present study.
} 
are vertical levels in the OGCM for each time step. Since this is a heavy load in terms of the computing costs, we use a moving average over several grid points in each horizontal direction (with equal weights as the simplest choice) as an approximation to the operator $P$. Using a moving average in the simple example in Fig. 2 yields results very similar to those for $P=L^{2} \nabla^{2}$ (not shown). Tests reveal that this approximate form of $P$ produces essentially the same results as the full operator in the OGCM, while only marginally increasing the computing costs. In the following, we call this method the smoothed semiprognostic method.

The second version of a semiprognostic method, which prevents damping of eddy activity, is realized by temporal averaging. We simply average the second term on the rhs of Eq. (2) for a certain period. Assuming that eddies in the ocean have preferred time scales less than the averaging period, we filter out their influence in the correction. On the other hand, short-term variability, that is, eddies, can then freely evolve in the OGCM, subject to the averaged correction term. We use simple 1-yr averages in the experiment discussed here to bypass effects of the seasonal cycle. In other words, the term $(1-\alpha)\left(\rho_{c}-\rho_{m}\right)$ is averaged over the first year of the integration and added to the model density $\rho_{m}$ in the second year, while further averaging the corresponding value during the second year, which is applied in the third year, and so forth. ${ }^{5}$ We call this version the mean semiprognostic method. We do not filter out possible small-scale data errors with the mean semiprognostic method as before with the smoothed semiprognostic method. However, a combination of smoothed and mean methods can certainly be used. We note that waves with periods less than a year (e.g., short baroclinic Rossby waves, coastal Kelvin waves, internal gravity waves) will be uneffected by the "mean" semiprognostic method, while the speed of waves with periods longer than a year (e.g., long baroclinic Rossby waves) will be reduced. Therefore, the mean method will act similar to the corrected-prognostic version for processes with time scales less than a year.

Last, the remaining caveat is drawback 3, a possible interaction of the semiprognostic method with topography, causing spurious up- and downwelling. One reason for this effect is that the climatological density may not imply a (geostrophically balanced) continuous boundary current. This can be a result of the interpolation and smoothing techniques used for the compilation of the climatology; for example, the slopes of

\footnotetext{
${ }^{5}$ Note that a "smoother" method can be obtained using a moving average over, e.g., the model time steps, of $1 \mathrm{yr}$. Using this moving average instead of the discrete annual averages would eliminate the otherwise sudden change in the correction at the end of each year but is, however, computationally very elaborate. Therefore, we stick to the former averaging method. Note also that a possible variation is the use of ensemble averages of the OGCM instead of temporal averages, but we do not explore this route in the present study.
}

the isopycnals across the boundary current might be reduced. This appears to be the case, for example, in the Gulf Stream of the climatology of Boyer and Levitus (1997), as our experiments suggest. To overcome this problem, we have tapered the parameter $\alpha$ near the boundaries to one, which means that the model becomes "locally prognostic" in calculating the boundary current system and semiprognostic in the interior. We call this method the tapered semiprognostic method. Note that it also appears possible to exclude, for example, the deep ocean from having an influence on the method by setting $\alpha$ to one below a certain depth since, there, observations might be less reliable.

In summary, to resolve difficulties $1-3$, we propose four modifications to the original semiprognostic method. Spatial averaging (smoothed method) or temporal averaging (mean method) of the correction term in the semiprognostic model is applied to reduce the damping influence on eddy activity. Possible data problems near the boundaries (or deep ocean) are accounted for there by relaxing the influence of the method (tapered method). Having done a spinup integration with one or a combination of these modified methods in a semiprognostic model, we proceed by diagnosing the correction term and applying it as a non-flow-interactive correction in the subsequent integration (corrected prognostic), which finally resolves the problem of modified physical properties.

\section{Results}

We have performed various experiments with the above-described semiprognostic methods applied to the eddy-permitting OGCM. In order to present the essential results of the experiments, we focus on a few key points in the circulation of the northern North Atlantic and discuss and compare selected prognostic and semiprognostic model results. These key points are the upperlevel flow of the North Atlantic Current around the Grand Banks southeast of Newfoundland and the flow of the Gulf Stream, from its separation from the shelf to the Grand Banks. We also compare important largescale features of the model, that is, northward heat transport, meridional overturning circulation, and distribution of upper-level eddy kinetic energy (EKE), to describe the model performance. However, to start, we discuss the surface air-sea heat flux as diagnosed in the prognostic models to point out possible systematic model errors.

\section{a. Prognostic models}

Figure 3a shows the annual mean heat flux as given by a 3-yr analysis period of the European Centre for Medium-Range Weather Forecasts (ECMWF) numerical weather forecast model (Barnier et al. 1995). According to this figure, the northern North Atlantic is losing heat almost everywhere with maximum heat loss 
a)

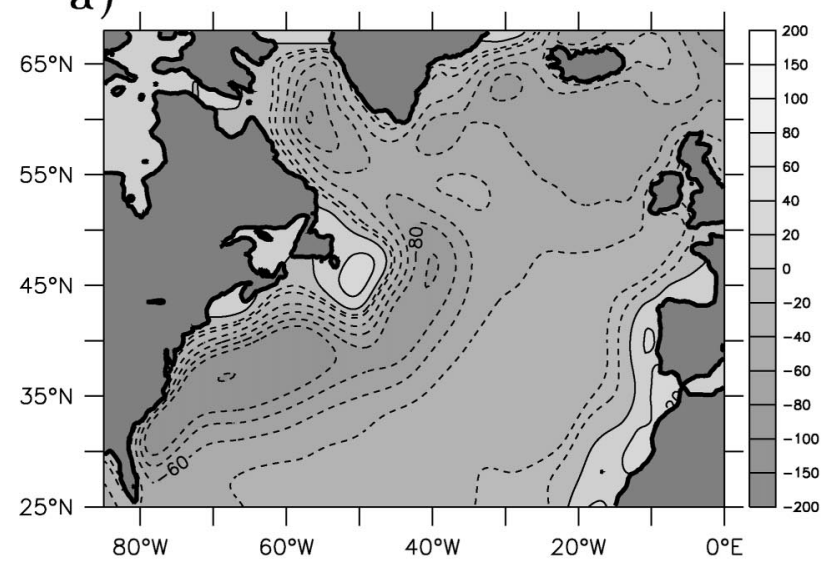

c)

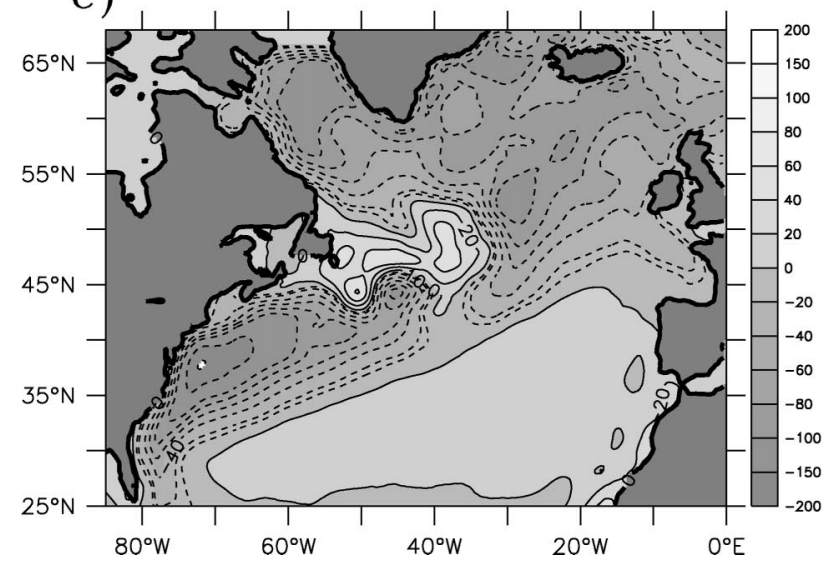

b)

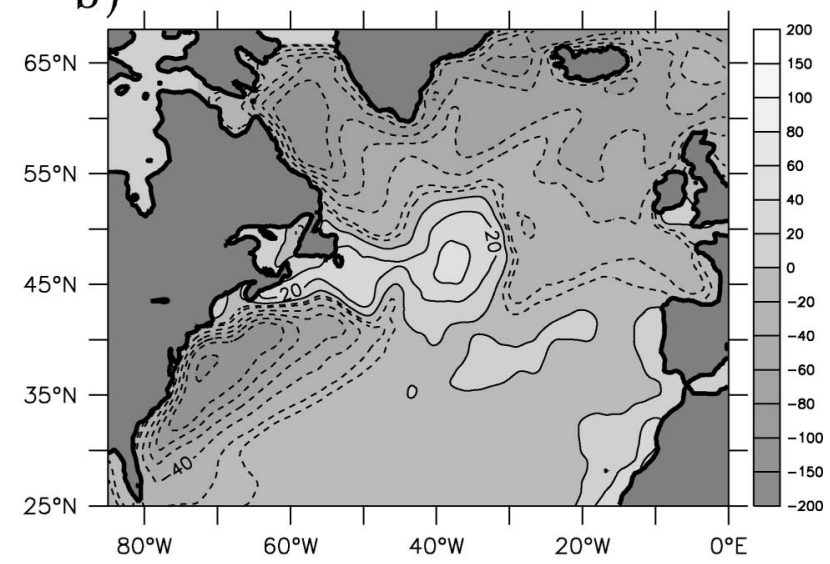

d)

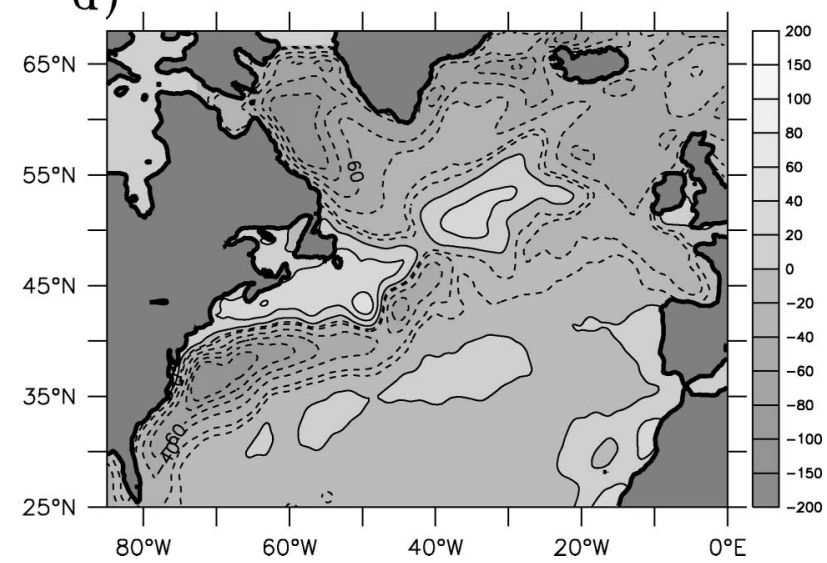

FIG. 3. (a) Annual mean net surface heat flux $\left(\mathrm{W} \mathrm{m}^{-2}\right)$ as given by the analysis of Barnier et al. (1995) driving the models as part of the surface (heat flux) boundary condition. Contour interval is $20 \mathrm{~W} \mathrm{~m}^{-2}$ in between and $50 \mathrm{~W} \mathrm{~m}^{-2}$ beyond the interval [ -100 , 100$] \mathrm{W} \mathrm{m}^{-2}$. (b) Three-year mean diagnosed heat flux in the (prognostic) FLAME eddy-permitting model. (c) As in (b) but for the DYNAMO model. (d) As in (b) but for the semiprognostic (FLAME) model. All data have been spatially smoothed with a ( $2^{\circ}$ half-width) boxcar window prior to plotting.

along the path of the Gulf Stream and the NAC southeast of Newfoundland, the central Labrador Sea, and Irminger Sea. Areas of (small) heat gain are located in the upwelling regions off the coast of North Africa and over the shallow Grand Banks east of Newfoundland. As for the DYNAMO models, the surface heat flux boundary condition for our OGCM is given as the (however, monthly varying) flux of Fig. 3a plus, following Barnier et al. (1995), a relaxation term, derived from linearized bulk formulas and accounting for a possible deviation of the modeled sea surface temperature from an observed, climatological one. Therefore, the flux in Fig. 3a may not coincide with the heat flux that actually enters the OGCM. In the case of a systematic model error, which shows up in the modeled SST, we expect a significant large-scale contribution in a longer-term average of the relaxation term. On the other hand, interpretation of the relaxation term must be careful since the surface forcing, either wind stress or buoyancy fluxes (or other components of the model setup), might also contain errors and show up (directly or indirectly by apparently false ocean model results) as well in the relaxation term.

Figure $3 \mathrm{~b}$ shows the diagnosed heat flux from the prognostic eddy-permitting model after the spinup, containing both the relaxation term and the net surface heat flux from ECMWF. It is clear from Fig. 3b that there is a significant large-scale contribution of the relaxation term along the path of the NAC around the Grand Banks, causing the most prominent difference from the ECMWF heat flux. We show in Fig. 3c the diagnosed heat flux from the $z$-level DYNAMO model as well. The DYNAMO model shows a similar contribution at the same location, pointing toward a common feature in the models.

Figure 4a shows the long-term mean observed temperature (Boyer and Levitus 1997) at 50-m depth around the Grand Banks. Evidently, there is a strong signature of the NAC flowing northward parallel to the Grand Banks, with warm water of subtropical origin to the right 

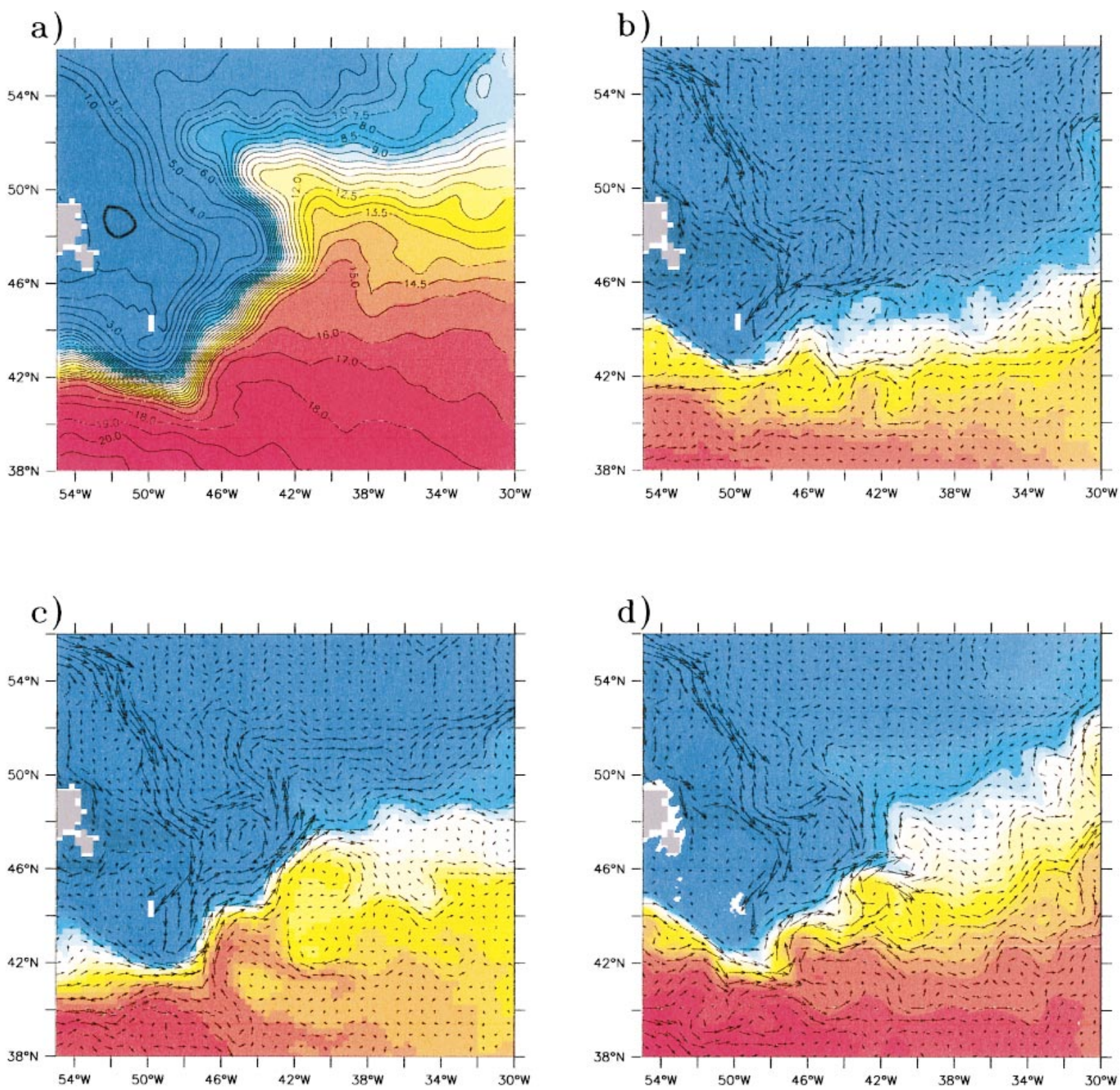

FIG. 4. (a) Annual mean climatological temperature taken from Boyer and Levitus (1997) at 50-m depth east of Newfoundland. Contour interval is $0.5 \mathrm{~K}$ in between and $1 \mathrm{~K}$ beyond the interval $\left[6^{\circ} \mathrm{C}, 15^{\circ} \mathrm{C}\right]$. (b) Three-year mean of temperature and velocities at the same depth as in the prognostic model. (c) As in (b) but for the semiprognostic model. (d) As in (b) but for a prognostic model with increased resolution $\left(1 / 12^{\circ}\right.$ instead of $\left.1 / 3^{\circ}\right)$. Every second vector is shown for the $1 / 3^{\circ}$ models and every eighth vector for the $1 / 12^{\circ}$ model.

and cold subpolar water to the left, until it penetrates eastward into the interior North Atlantic at about $52^{\circ} \mathrm{N}$. Figure $4 \mathrm{~b}$ shows mean temperature and velocities at the same depth in the prognostic model. Clearly, there is almost no northward flow east of the Grand Banks in the prognostic model, which leads to too-cold water in comparison with the observations north of about $46^{\circ} \mathrm{N}$ and, in consequence, to the large contribution to the relaxation term of the surface heat flux. Therefore, we conclude that the contribution of the relaxation term in this region shows a significant systematic error of the OGCM. Second, we can assume that this is not just an artifact of our specific model, but a common problem in this class of eddy-permitting models of the North Atlantic, as previously noted by Böning et al. (1996) and by Willebrand et al. (2001) for the DYNAMO (level) showing a similar deficiency. Coupling such an ocean model to an atmospheric general circulation model would likely raise the need for a flux correction at this location. 
a)

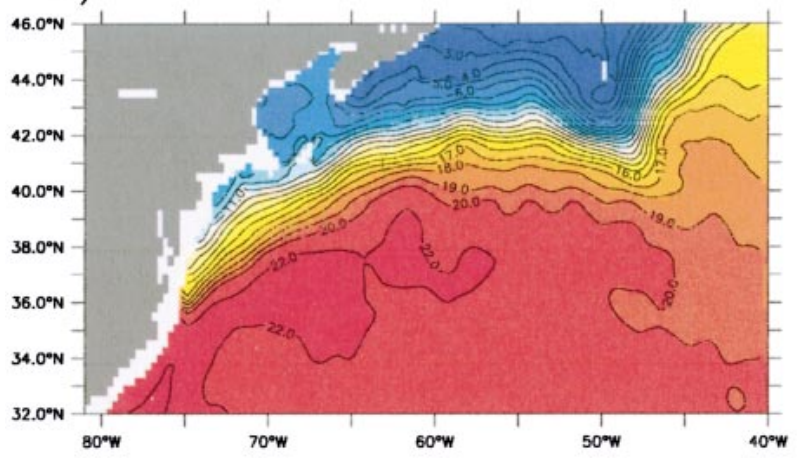

c)

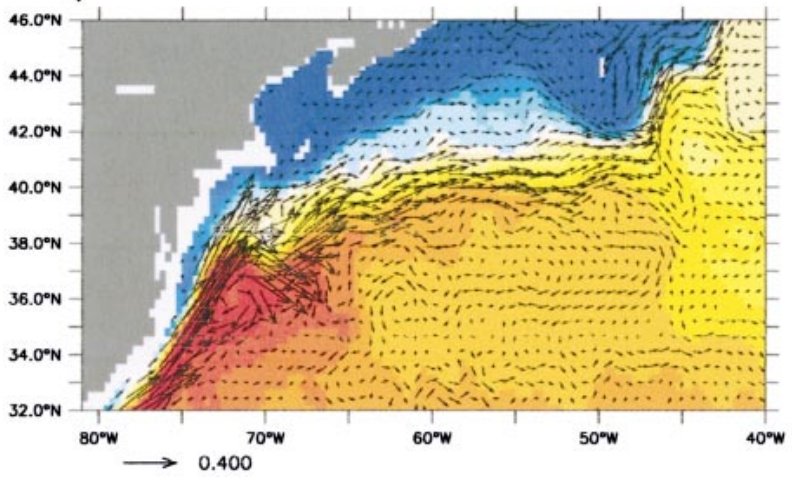

b)



d)

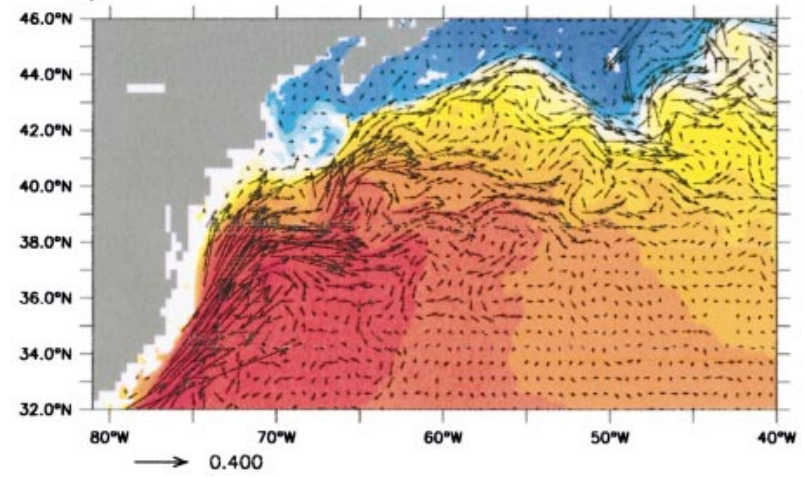

Fig. 5. (a) Annual mean climatological temperature taken from Boyer and Levitus (1997) at 50-m depth. Contour interval is 1 K in the interval $\left[-2^{\circ} \mathrm{C}, 20^{\circ} \mathrm{C}\right]$ and $2 \mathrm{~K}$ beyond. (b) Three-year mean of temperature and velocities at the same depth in the prognostic model. (c) As in (b) but for the semiprognostic model. (d) As in (b) but for a prognostic model with increased resolution $\left(1 / 12^{\circ}\right.$ instead of $\left.1 / 3^{\circ}\right)$. Every second vector is shown for the $1 / 3^{\circ}$ models and every eighth vector for the $1 / 12^{\circ}$ model.

\section{b. Original semiprognostic model}

In the semiprognostic models the flow around the Grand Banks is more realistic. Figure 4c shows mean temperature and velocities at 50-m depth in an experiment applying the original semiprognostic method in the eddy-permitting OGCM. Water north of $46^{\circ} \mathrm{N}$ is up to $8^{\circ} \mathrm{C}$ warmer than in the prognostic version. In fact, the circulation is now becoming similar to model solutions with drastically increased horizontal resolution. Figure $4 \mathrm{~d}$ shows mean temperature and velocities for the eddy-resolving model in this region, agreeing surprisingly well with the semiprognostic model. The northward migration of the northern boundary of subtropical water east of the Grand Banks coming along with increased horizontal resolution, as observed here for the FLAME models, is also reported by Smith et al. (2000, their Figs. 9b and 9c). Thus, for a more realistic simulation of this region, that is, the correct flow pattern of the NAC, it appears necessary to use very high horizontal resolution in the OGCM, which is presently too costly for use in a coupled modeling system. The semiprognostic model produces a similar degree of realism using much fewer resources, although it is still too cold in comparison with the observations, as in the eddyresolving versions. However, carrying over this im- provement to a corrected-prognostic version of the model would be of benefit.

Farther upstream of the NAC, we meet another region in which eddy-permitting North Atlantic models usually fail to simulate realistic flow patterns and water mass structure. This is the Gulf Stream from its point of separation from the shelf near Cape Hatteras to the Grand Banks. Figure 5 shows in this region the observed temperature (Fig. 5a) and modeled temperature and velocity in the prognostic model (Fig. 5b), the semiprognostic model (Fig. 5c), and the $1 / 12^{\circ}$ model (Fig. 5d). We see for the prognostic eddy-permitting model as well as for the eddy-resolving model too-warm water at the northern flank of the Gulf Stream. Furthermore, there are strong recirculation cells near the separation from the shelf. The latitude of separation appears too far to the north. In the interior, the stream does not show a jetlike structure, but, in contrast, several flow paths, most of them too far to the north, leading apparently to the toowarm water north of the Gulf Stream. This is in agreement with results from many previous model solutions and in contrast to observations, as described by Dengg et al. (1996). Note, however, that while the FLAME eddy-resolving model fails to simulate a realistic Gulf Stream, other high-resolution OGCMs are able to pro- 


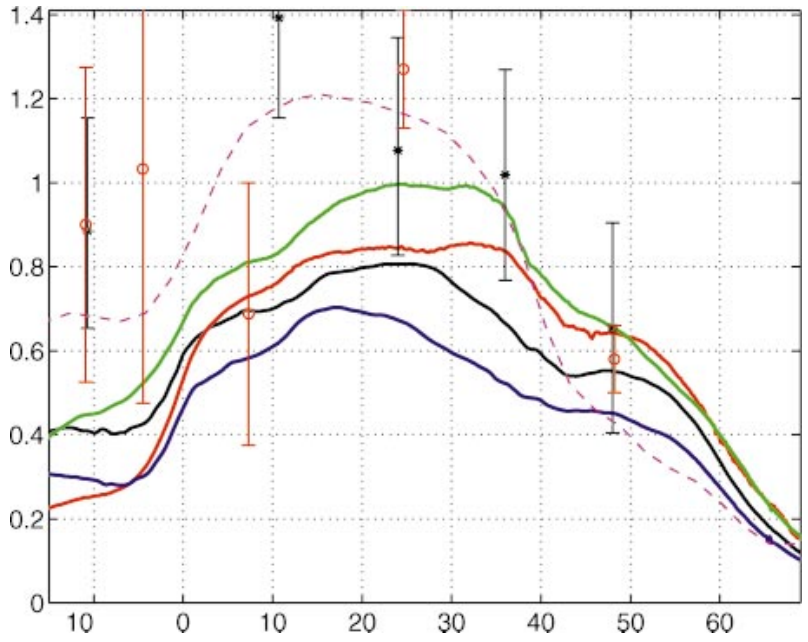

FIG. 6. Three-year averages of northward heat transport (PW) in the eddy-permitting prognostic model (black, thick line), the DYNAMO (red, thick line), the eddy-resolving model (green, thick line), and the semiprognostic model (blue, thick line). Also shown are observational estimates of oceanic heat transports given by MacDonald and Wunsch (1996) (black circles with error bars), by Ganachaud and Wunsch (2000) (red circles with error bars), and by Trenberth and Caron (2001) (dashed, magenta line).

duce realistic simulations (Smith et al. 2000; Paiva et al. 1999).

In the semiprognostic model, the situation is again more realistic than in the prognostic version; there is a distinct jetlike flow to the Grand Banks. Although we see still an unrealistic recirculation cell near Cape Hatteras, it is much smaller in amplitude when compared with the prognostic model. Furthermore, temperatures north of the flank of the Gulf Stream are now several kelvins cooler than in the prognostic model and in much better agreement with the observations.

The improvements, shown by the semiprognostic model with respect to the prognostic model, in simulating more realistically some of the major flow patterns in the North Atlantic give some hope that the spurious anomalies in the surface heat fluxes due to systematic model errors are now reduced. It turns out, however, that the improvements are not as large as expected. Figure $3 \mathrm{~d}$ shows the diagnosed heat flux for the semiprognostic model. Comparison with Fig. $3 b$ shows that we now find heat loss east of the Grand Banks with maximum amplitude of $60 \mathrm{~W} \mathrm{~m}^{-2}$, but still less than in the ECMWF heat flux where the heat loss reaches $100 \mathrm{~W}$ $\mathrm{m}^{-2}$ and covers a broader region. Moreover, we see also a region of heat gain north of $50^{\circ} \mathrm{N}$.

This result points toward too little northward transport of warm water in the semiprognostic model. Figure 6 shows the mean northward heat transport for the prognostic, the semiprognostic, the DYNAMO, and the eddy-resolving models. While heat transports in the prognostic, eddy-permitting FLAME and DYNAMO models are similar, ${ }^{6}$ the eddy-resolving model shows in general more, and the semiprognostic model less, northward heat transport. Also shown are observational estimates derived from hydrographic data by MacDonald and Wunsch (1996) and Ganachaud and Wunsch (2000) and derived from atmospheric data by Trenberth and Caron (2001). Since the observations show a large spread within approximately $10^{\circ}$ of the equator and our main focus is on the midlatitude North Atlantic, we omit here a discussion of possible model (forcing, data) errors in the tropical Atlantic. However, it is evident that the eddy-permitting models show a bias in carrying too little heat northward in comparison with the observations, while the eddy-resolving model shows stronger northward heat transport. Note that the enhanced heat transport in the eddy-resolving model coming along with increased resolution is in agreement with the model results discussed by Smith et al. (2000). However, more realistic flow patterns and watermass characteristics in the semiprognostic model do not lead to an effect similar to that from increased resolution; in contrast, the bias to unrealistic low northward heat transport is enhanced in the semiprognostic model.

The major mechanism to transport heat northward in the North Atlantic is the meridional overturning circulation (Böning et al. 1995). Figure 7 shows the meridional streamfunction for the prognostic (Fig. 7a) and the semiprognostic model (Fig. 7b). In the prognostic model, we see around $45^{\circ} \mathrm{N}$ maximum northward volume transport in the upper $1000 \mathrm{~m}$ of about $14 \mathrm{~Sv}$ ( Sv $\left.\equiv 10^{6} \mathrm{~m}^{3} \mathrm{~s}^{-1}\right)$. A similar amount is transported in the semiprognostic model at this latitude, but strong upwelling between $30^{\circ}$ and $35^{\circ} \mathrm{N}$ reduces the northward volume transport south of $30^{\circ} \mathrm{N}$ to about $8-9 \mathrm{~Sv}$ in the semiprognostic model. In the prognostic model, the upwelling is not as strong: 10-11 Sv are still carried northward south of $30^{\circ} \mathrm{N}$. The strengthened shortcut of the overturning circulation by the enhanced upwelling in midlatitudes in the semiprognostic model in comparison with the prognostic model leads apparently to the bias toward low heat transport.

The semiprognostic model and, to a lesser extent, the prognostic model both suffer from a long-known model artifact, the so-called Veronis effect. As first described by Veronis (1975), horizontal diffusion in OGCMs generates diapycnal buoyancy fluxes in the presence of steep slopes of isopycnals. Strongly inclined isopycnals can be found in western boundary currents and are most prominent in the Gulf Stream. Here, the diffusive diapycnal buoyancy fluxes across the front of the current are balanced by vertical advective transports, showing

\footnotetext{
${ }^{6}$ The (maybe spurious) heat uptake between about $30^{\circ}$ and $40^{\circ} \mathrm{N}$ in DYNAMO in contrast to the (more reasonable) heat loss in FLAME is most likely due to different mixed layer schemes. There was none in DYNAMO, while FLAME uses a total kinetic energy (TKE) scheme. See also the difference in heat fluxes in the eastern, subtropical North Atlantic in Figs. $3 b$ and $3 c$.
} 
a)

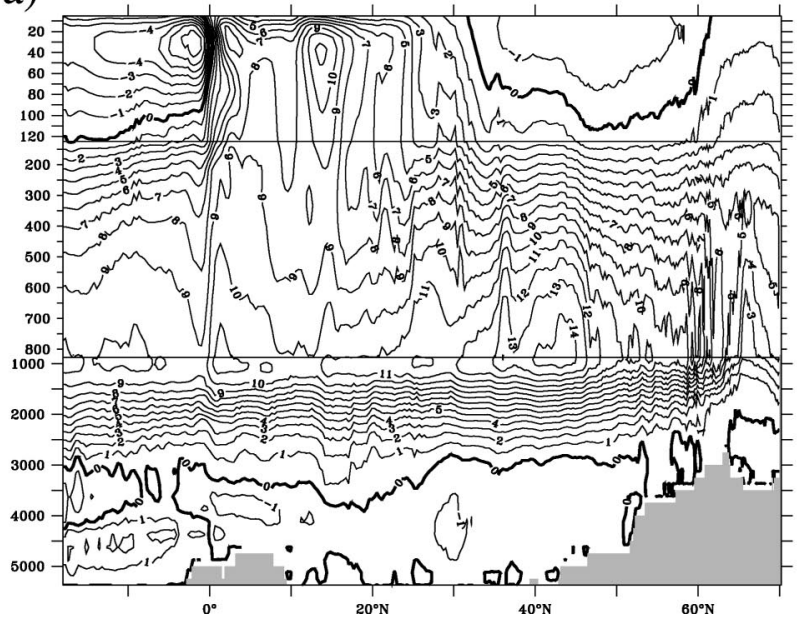

b)

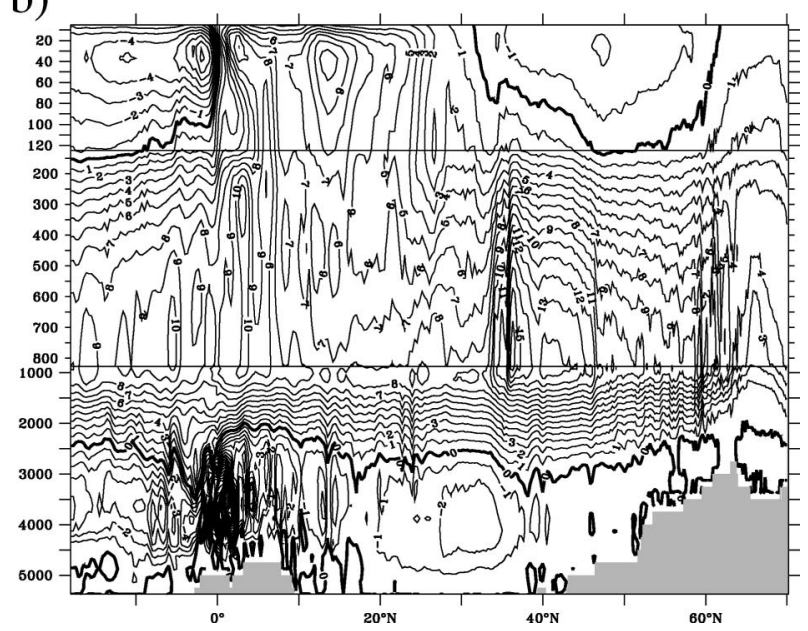

FIG. 7. Three-year averages of the meridional streamfunction (Sv) for (a) the eddy-permitting prognostic model and (b) the semiprognostic model. Contour interval is $1 \mathrm{~Sv}$. Note the stretched vertical axis above 800 and $120 \mathrm{~m}$.

up as the spurious (cold) upwelling in the meridional streamfunction and reducing the northward heat transport in consequence. This model artifact can be effectively reduced by the use of isopycnal diffusion, that is, tracer diffusion oriented along isopycnals (Böning et al. 1995; Griffies 1998). Apparently, the (small, flow interactive) implicit, numerical diffusion of the advection scheme (Quicker) used for the eddy-permitting OGCM acts more like horizontal diffusion instead of isopycnal diffusion, leading in the prognostic model to the upwelling south of $35^{\circ} \mathrm{N}$ of about $3 \mathrm{~Sv}$. We note here in passing that in a version of the same eddy-permitting model using the (traditional) second-order centered differences advection scheme together with (explicit) isopycnal diffusion, the upwelling effect is reduced in comparison with the version with Quicker and without explicit diffusion, coming along with a stronger overturning circulation and with increased northward heat transport. On the other hand, the "isopycnal" version shows almost entirely suppressed eddy activity and less realistic flow of the Gulf Stream and NAC (both not shown) in comparison with the version with the Quicker advection scheme. However, we make no further use of the isopycnal model version (neither prognostic nor semiprognostic) in the present paper.

In the semiprognostic model, as shown in Fig. 7b, the Veronis effect is enhanced. We may have indirectly changed the diffusive buoyancy transports with the method by changing the advective flow and therefore the implicit, numerical diffusion. However, we regard this diffusive effect as minor in comparison with vertical velocities that we might generate using the semiprognostic method near boundaries with steep isopycnals as in the Gulf Stream region. Here, the climatological density $\rho_{c}$ that we use apparently contains information about the boundary current transport [caveat 3 in section $2 \mathrm{c}$ ] that is inconsistent with the model. The resulting in- consistency is then accounted for by spurious up- (or down-) welling near the western boundary (not shown), leading to the enhanced Veronis effect as seen in Fig. $7 b$. Note that using the smoothed and/or tapered semiprognostic methods, the (inconsistent) information about the (small scale) slopes of the boundary current is essentially excluded from having influence, with the effect of reduced spurious upwelling, as we shall show in section $3 \mathrm{c}$, confirming this interpretation.

In contrast to the prognostic model, there are also strong recirculation cells in the meridional streamfunction of the semiprognostic model around the equator below about $2500 \mathrm{~m}$ with a maximum at about $4000 \mathrm{~m}$. The reason is another interaction between topography and the semiprognostic method. The topography of the model allows ${ }^{7}$ through a small gap in the Mid-Atlantic Ridge system at the equator (the model representation of the Vema and Romanche Fracture Zones), for a crossequatorial flow east of $25^{\circ} \mathrm{W}$ below $4000 \mathrm{~m}$. In the climatology of Boyer and Levitus (1997) we find in the eastern equatorial region in the Atlantic below $4000 \mathrm{~m}$ cool and fresh water south of the equator (resembling Antarctic Bottom Water) and relatively warm and saline water north of the equator (more akin to North Atlantic Deep Water). Apparently, the modeled (northward) transport through the Vema and Romanche Fracture Zones is unrealistic: both prognostic and semiprognostic models deviate from the observations; that is, they show cooler and fresher water than in the observations on the northern side of the gap and warmer and more saline water on the southern side (not shown), pointing toward

\footnotetext{
${ }^{7}$ In fact, the topography of the eddy-permitting FLAME model was manipulated by hand after discretization to allow for this throughflow (Kröger 2001). Similar hand-tuning was applied at several other locations, e.g., the Denmark and Florida Straits.
} 
a too-strong deep cross-equatorial flow into the eastern North Atlantic.

The semiprognostic method generates corrective pressure gradients in the deep eastern equatorial region due to large deviations from the climatology that, however, do not lead to an improvement in the model density since transports across the Vema and Romanche Fracture Zones are apparently unchanged. The corrective pressure gradients are largest at the narrow throughflow point at the equator, leading to the spurious recirculation cells between 2500 and $4500 \mathrm{~m}$ in Fig. 7b. Clearly, this result points to another potential problem of the semiprognostic model. A simple way to resolve this problem is to manipulate the topography in this region and to inhibit the artificially strong throughflow. However, we have not made such an attempt since the modified versions of the method take care of this problem, as we shall show below.

In summary, we see that the semiprognostic model performs well in improving the simulation of major advective pathways and, consequently, watermass structures of the North Atlantic, but we encounter problems (in addition to reduced wave speed and damped mesoscale activity). First, there is stronger upwelling in the Gulf Stream region in comparison with the prognostic model, leading to a reduction of northward heat transport. Second, there are potential problems near the topography. By spurious transports through artificial gaps, large corrective pressure gradients can build up, which are unable to improve the solution. We show in the next section that the modified semiprognostic methods are able to resolve these problems.

\section{c. Smoothed, mean, and tapered semiprognostic models}

We discuss in this section results from experiments with the modified semiprognostic methods. The main experiments in this section are the following:

- experiment MEAN, in which the mean semiprognostic method is applied in the same manner as explained in section $2 \mathrm{c}$;

- experiment SMOOTH, applying both the smoothed and tapered semiprognostic method; the moving average involves 10 grid points in each direction (equal weights), and, within 6 grid points of distance from land, the parameter $\alpha$ is set to 1 (and to 0.5 everywhere else);

- experiment MEAN + SMOOTH, in which MEAN and SMOOTH are combined; that is, the averaged correction of the mean method is smoothed and tapered as the instantaneous correction in SMOOTH; and

- experiment MEAN + SMOOTH-800, repeating MEAN + SMOOTH with the difference that here the moving average involves only five grid points and $\alpha$ is tapered within three grid points of distance from land. Moreover, $\alpha$ is set to 1 below $800 \mathrm{~m}$.

We remind the reader that the spinup and analysis procedures and other details are discussed in section $2 \mathrm{a}$ and that in all cases the methods are applied to the eddypermitting FLAME model.

To start, we confirm that the mean and smoothed methods reduce the damping influence of the original semiprognostic method on eddy activity. Figure $8 \mathrm{a}$ shows eddy kinetic energy at 50-m depth in the prognostic eddy-permitting OGCM. Maxima of EKE show up in the Gulf Stream region and along the path of the NAC, with smaller local maxima in the Labrador Sea and the Irminger Sea. It must be noted that there is a bias of too-low EKE in eddy-permitting OGCMs in midlatitudes (Smith et al. 2000; Eden and Böning 2002); eddy-resolving OGCMs and observational estimates show order of magnitude higher levels. The reason is insufficient horizontal resolution to capture the bulk of the essential hydrodynamic instability processes in eddy-permitting models. On the other hand, the horizontal distribution of near-surface EKE in eddy-permitting OGCMs is in general agreement with observational estimates (Stammer et al. 1996).

As expected, EKE is damped in the model using the original semiprognostic method, as Fig. $8 \mathrm{~b}$ reveals. The basinwide average of EKE at 50-m depth is $63 \times 10^{-4}$ and $36 \times 10^{-4} \mathrm{~m}^{2} \mathrm{~s}^{-2}$ for the prognostic model and the model utilizing the original semiprognostic method, respectively. On average, upper-level EKE is thus reduced by one-half with the original semiprognostic method. However, the mean and smoothed versions of the method recover (and exceed) the level of EKE in the prognostic version. Figures 8c and 8d show the corresponding results from MEAN and SMOOTH. In both figures, maxima of EKE are similar to that of the prognostic model, showing the success of both methods. The basin averages of EKE at 50-m depth are $84 \times 10^{-4} \mathrm{~m}^{2} \mathrm{~s}^{-2}$ for MEAN ${ }^{8}$ and $68 \times 10^{-4} \mathrm{~m}^{2} \mathrm{~s}^{-2}$ for SMOOTH. The reason for the excess of EKE in MEAN is that MEAN shows enhanced values of EKE compared to the prognostic model in the more quiescent regions, like the eastern subtropical North Atlantic. This is not the case for SMOOTH, but only minor differences in the maxima of EKE show up in comparison with MEAN. Furthermore, the mean and smooth methods clearly lead to a northward migration of maximum EKE east of Newfoundland, pointing toward more realistic flow of the $\mathrm{NAC}$ in this region.

EKE in MEAN + SMOOTH (not shown) is similar to SMOOTH; the basin average of EKE at 50-m depth is $69 \times 10^{-4} \mathrm{~m}^{2} \mathrm{~s}^{-2}$, while EKE in MEAN +

\footnotetext{
${ }^{8}$ Note that we get similar (or even higher) ratios of EKE in MEAN in comparison with the other experiments, restricting the calculation of EKE to the second half of each year of the analysis period, thus excluding the barotropic and inertial adjustments to the changing correction term in MEAN.
} 
a)

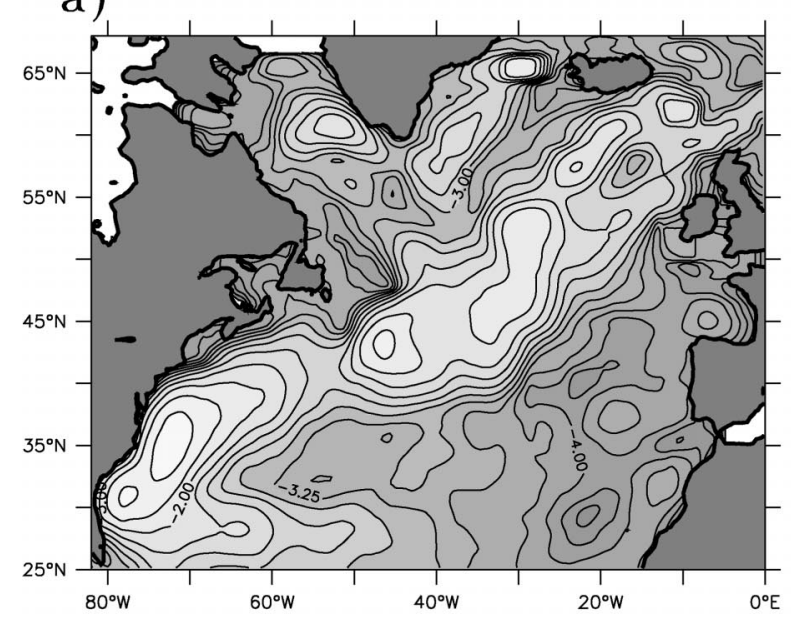

c)

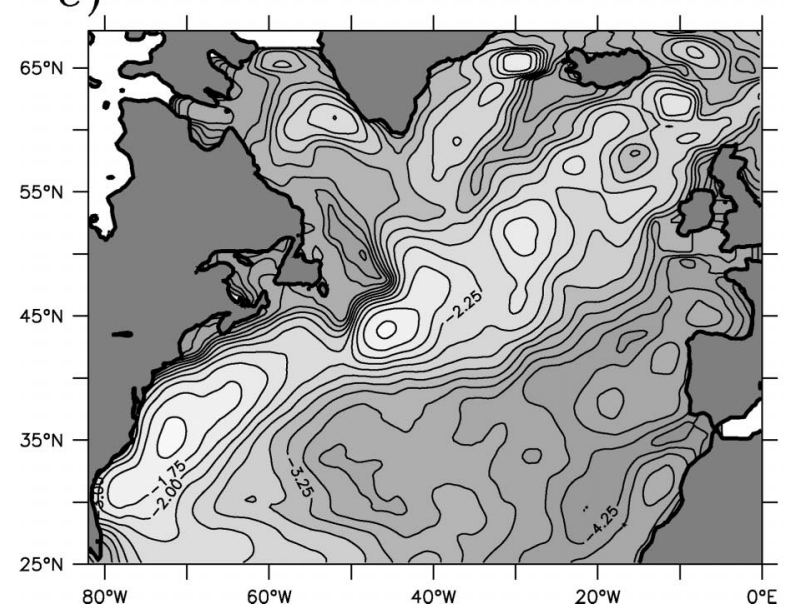

b)

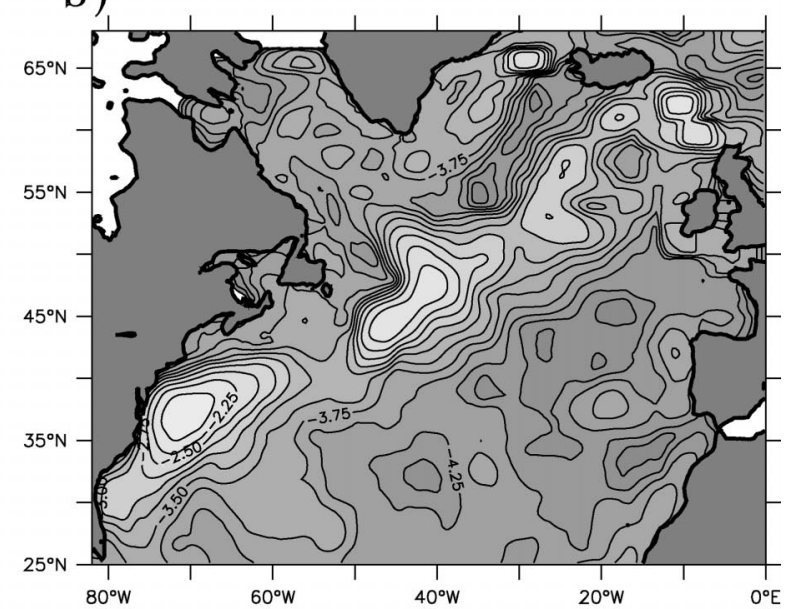

d)

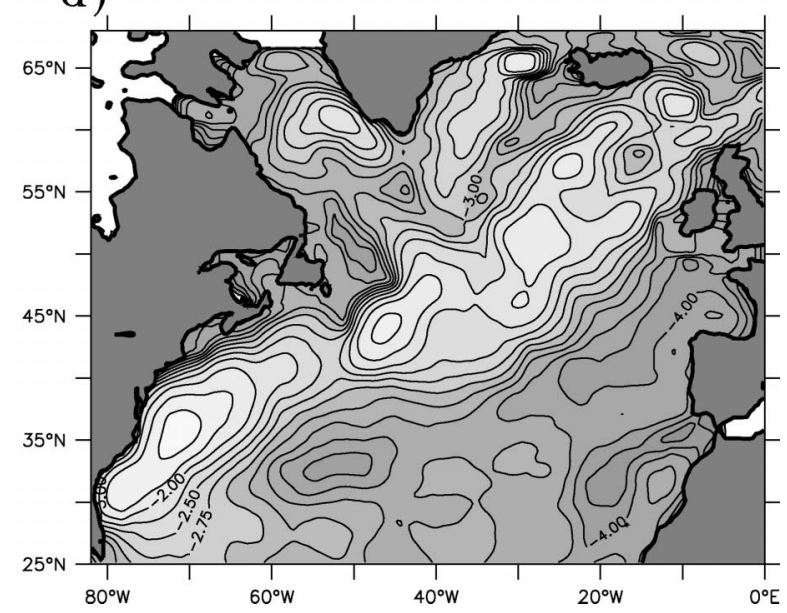

FIG. 8. EKE at 50-m depth $\left(\mathrm{m}^{2} \mathrm{~s}^{2}\right)$. Shown is the logarithm of EKE; i.e., values at contour lines are powers of 10; contour interval is 0.25. (a) EKE in the prognostic eddy-permitting model, and (b)-(d) EKE in semiprognostic models using the original method, the mean (expt MEAN) method, and the smoothed and tapered (expt SMOOTH) method, respectively. EKE was calculated from velocity deviations from seasonal means and averaged over the 3 -yr analysis period. The data have been spatially smoothed with a $\left(2^{\circ}\right.$ half-width) boxcar window prior to plotting.

SMOOTH-800 (where we have reduced the length scale and, therefore, the influence of the moving average) is more akin to MEAN, with a basin-average EKE of 73 $\times 10^{-4} \mathrm{~m}^{2} \mathrm{~s}^{-2}$. The differences suggest that the temporal averaging in MEAN is superior in preserving EKE in a semiprognostic model, as compared with the spatial averaging. However, both methods are successful in that respect; that is, the level of EKE in the prognostic model is at least recovered in all experiments with the modified semiprognostic methods.

Most of the improvements of the original semiprognostic method with respect to flow patterns in the Gulf Stream and NAC are carried over to the mean, smoothed, and tapered methods. Figure 9a exemplifies the flow of the NAC around the Grand Banks in the experiments with the modified semiprognostic method. The figure shows results from MEAN, and the other experiments are similar. The temperature distribution and flow pattern are similar to the results of the original method (Fig. 4c) showing that we can achieve the same effect in this region with the modified versions as with the original version without affecting the small-scale variability. Note that we even see slightly warmer water north of about $48^{\circ} \mathrm{N}$ (more similar to the eddyresolving model), most likely due to enhanced mixing across the front by mesoscale variability in the modified versions.

Figure $9 \mathrm{~b}$ shows the simulation in MEAN for the Gulf Stream region. In contrast to the original semiprognostic model, we now see an almost vanishing spurious recirculation cell at the separation point and, as before, a jetlike flow to the southern tip of the Grand Banks. The temperature distribution is again in good agreement with Boyer and Levitus (1997). The success 
a)

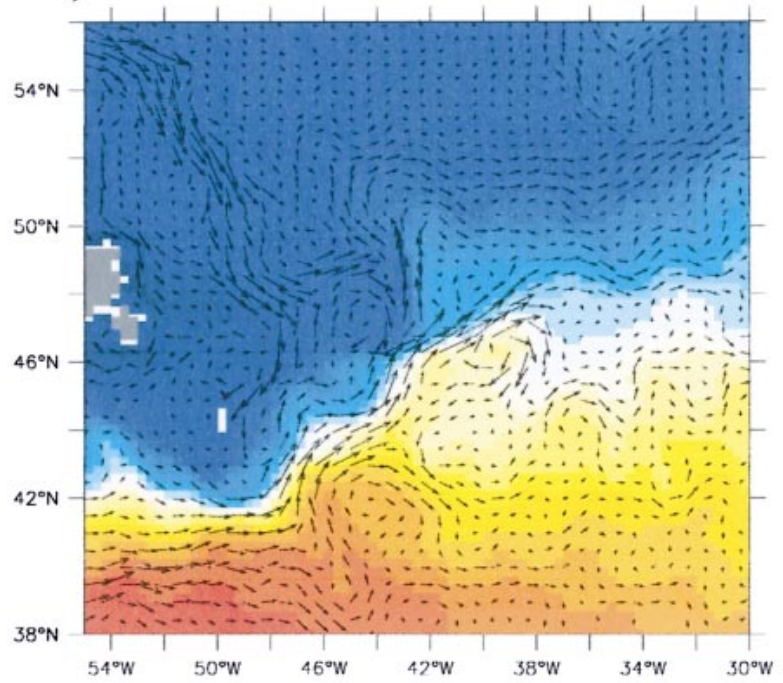

d)

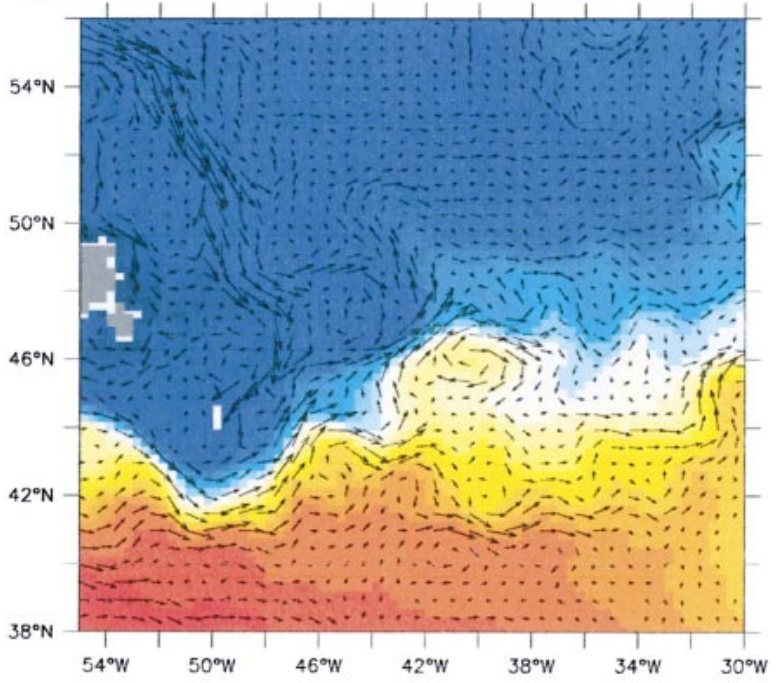

b)

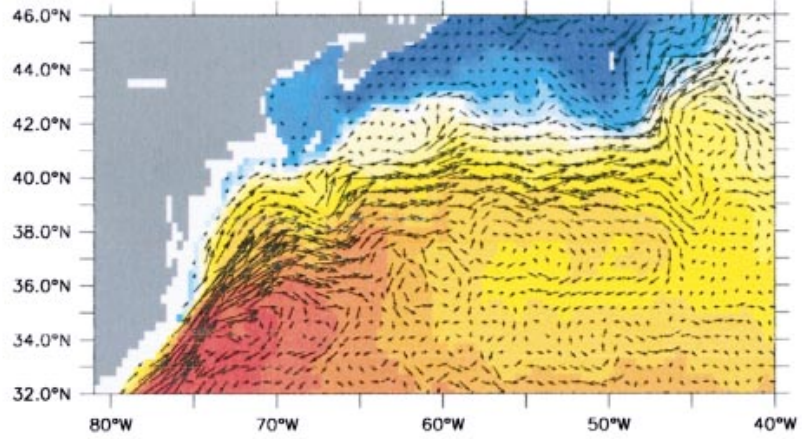

c)

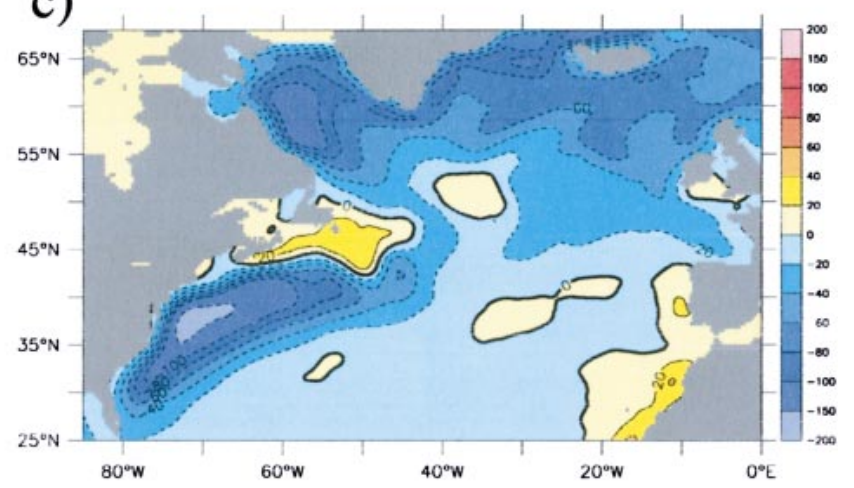

e)
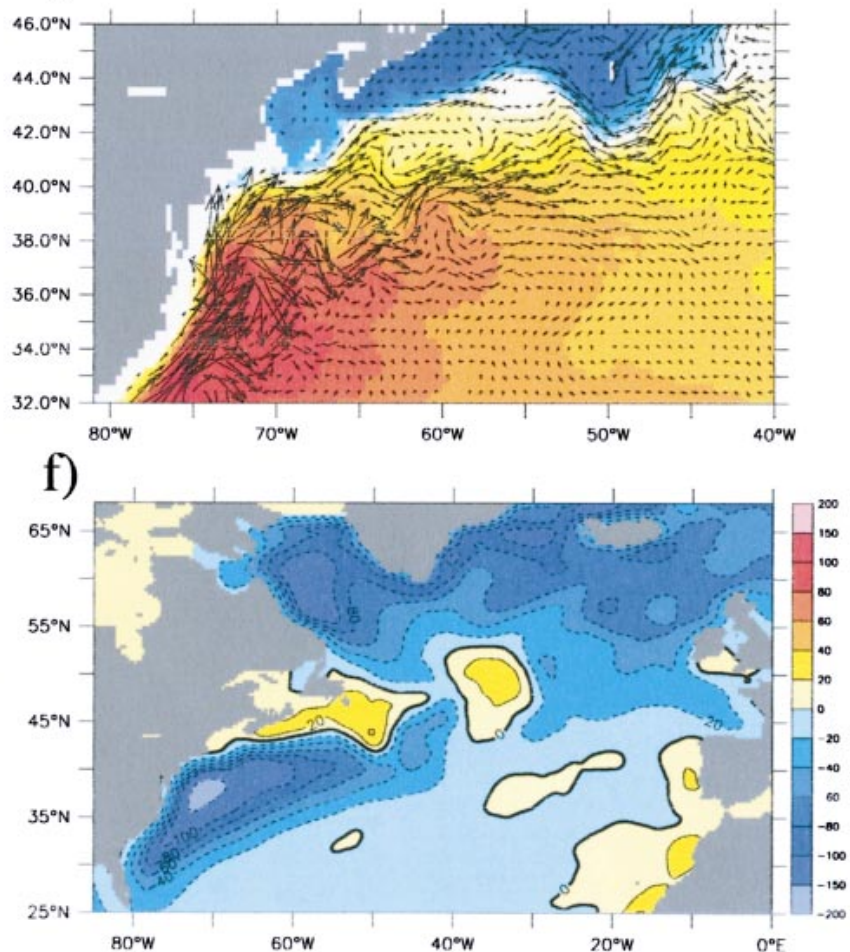

FIG. 9. (a), (b) Three-year mean of temperature and velocities at 50-m depth in the mean semiprognostic model (MEAN). Contour intervals etc. are the same as for Figs. 4 and 5, respectively. (c) Three-year mean diagnosed heat flux (similar to Fig. 3) for the mean and tapered semiprognostic model (MEAN + SMOOTH-800). (d), (e) As in (a), (b) but for the corrected prognostic model experiment MEAN + SMOOTH-800-CORR. (f) As in (c) but for MEAN + SMOOTH-800-CORR. 
of the mean semiprognostic method in eliminating the recirculation cells can be understood in the following way. If such a recirculation cell is developing in the course of a year, the averaged correction is account in the next year for that deviation of model density from the climatological density, that is, by redirecting the flow, which will tend to reduce the potential energy, stored in the spurious cell. Thus, on average, the mean semiprognostic method tends to damp out spurious, standing eddies. It must be noted, however, that in the other experiments with modified versions the simulation with respect to the separation is inferior to MEAN; that is, in the tapered experiments we see stronger recirculation cells than in MEAN. Clearly, the reason is the missing (in SMOOTH) or reduced (in MEAN + SMOOTH) damping influence on standing eddies of the mean semiprognostic method. However, there is still, as in MEAN, a jetlike flow of the Gulf Stream in the interior after separation (not shown) in the other experiments.

Flow patterns and watermass characteristics of MEAN + SMOOTH-800 are almost identical to those in MEAN + SMOOTH (not shown), revealing two results. First, sensitivity to the length scale of the smoothing operator $P$ (moving average) is apparently small, except for EKE, as discussed above. Second, using density in the upper ocean (roughly above the thermocline) only for the semiprognostic method appears to be sufficient to obtain the same benefits. In contrast, an experiment (not shown) applying the mean semiprognostic method only in the upper $200 \mathrm{~m}$ shows almost no difference from the original, prognostic model, while an experiment (not shown) applying the mean method from 200 to $800 \mathrm{~m}$ recovers almost all of the results of MEAN. The likely reason is that the greatest shear of geostrophic velocities; that is, the flow component, which is directly influenced by the semiprognostic method, can be seen in the thermocline.

Figure 9c shows the diagnosed surface heat flux from MEAN + SMOOTH-800. We see that the strong heat gain east of Newfoundland, as found in the prognostic eddy-permitting models, has now almost disappeared. Clearly, the situation with respect to this model artifact has also improved in comparison with the original semiprognostic model. MEAN, SMOOTH, and MEAN + SMOOTH are all very similar to Fig. 9c, confirming the benefit of the modified versions.

However, differences in the experiments with the modified methods show up in the meridional heat transport shown in Fig. 10a. In SMOOTH, MEAN + SMOOTH, and MEAN + SMOOTH-800 there is an increase in heat transport when compared with the prognostic model, which is most pronounced, about $0.1 \mathrm{PW}$ at maximum, in MEAN + SMOOTH and MEAN + SMOOTH-800. MEAN shows less heat transport; however, it is still more than that from using the original semiprognostic method. The reduced heat transport in MEAN comes along with weaker overturning circula- tion south of $30^{\circ} \mathrm{N}$ due to stronger upwelling near $35^{\circ} \mathrm{N}$ (not shown) when compared with the prognostic model; the same deficiency as we have seen for the original semiprognostic method is here, however, slightly reduced.

In contrast, the other (smoothed and tapered) experiments do not show this deficiency anymore. In MEAN + SMOOTH-800 the maximum northward volume transport south of $30^{\circ} \mathrm{N}$ is enhanced by $1 \mathrm{~Sv}$ as compared with the prognostic model. The upwelling (Veronis effect) is reduced, although still present, as Fig. 10b reveals. The corresponding streamfunction for MEAN + SMOOTH (not shown) is very similar to the one in MEAN + SMOOTH-800; in SMOOTH (also not shown) its shape is similar to that in Fig. 10b, but with reduced (about $1 \mathrm{~Sv}$ ) maximal amplitude. The mean method still includes all small-scale information in the climatological density, in particular the boundary current structure, while in the smoothed and tapered methods these structures are effectively excluded. We can conclude that it is the spatial smoothing and tapering of the semiprognostic correction that resolves the spurious upwelling in the Gulf Stream region (Veronis effect) as seen for the original method (and to a lesser extent in MEAN).

In summary, with respect to EKE both modified semiprognostic versions (mean and smoothed) perform better than the original method with larger EKE for the mean method, even larger than in the prognostic version. Improvements in flow patterns and watermass characteristics as given by the original method are carried over to the modified model, or are even outperformed. The same benefits can be obtained by applying the method in and above the thermocline only. Problems discovered for the original semiprognostic method involving interaction with topography are resolved with the modified versions. The spurious upwelling near the Gulf Stream separation is reduced using the smoothed and tapered methods, with the effect of enhanced northward heat transport. However, application of the mean method, without combination with the other methods, appears to be not as successful in this respect.

\section{d. Corrected-prognostic models}

Having now established the modified semiprognostic methods, we finally present results from corrected-prognostic models in this section. The semiprognostic correction is diagnosed and averaged in the analysis period of the experiments described above in section 3c. This diagnosed, no longer flow-interactive, correction is applied to the model in the subsequent integration. All corrected-prognostic model experiments presented here start from the end of the analysis period of the corresponding semiprognostic models and last for 7 years, to allow for another period of baroclinic adjustment. Results are shown as averages over the last three years of that integration. We add the suffix "-CORR" to the 
a)

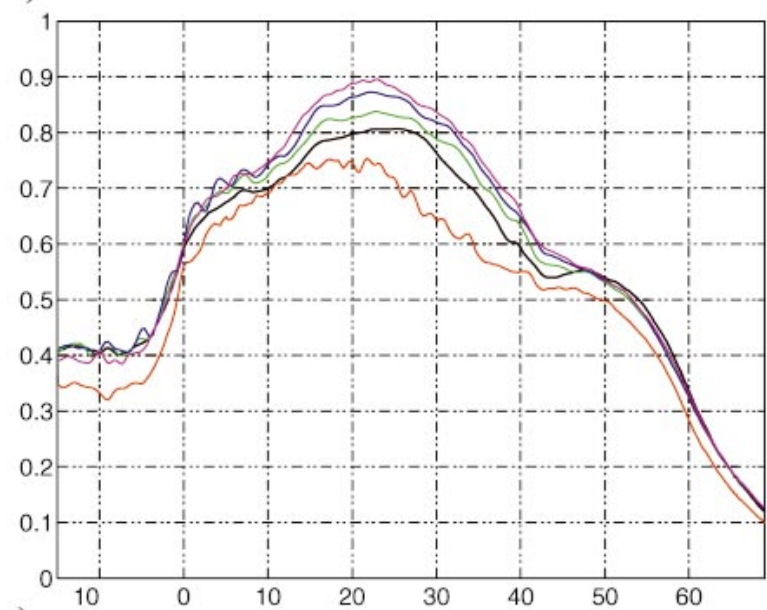

c)

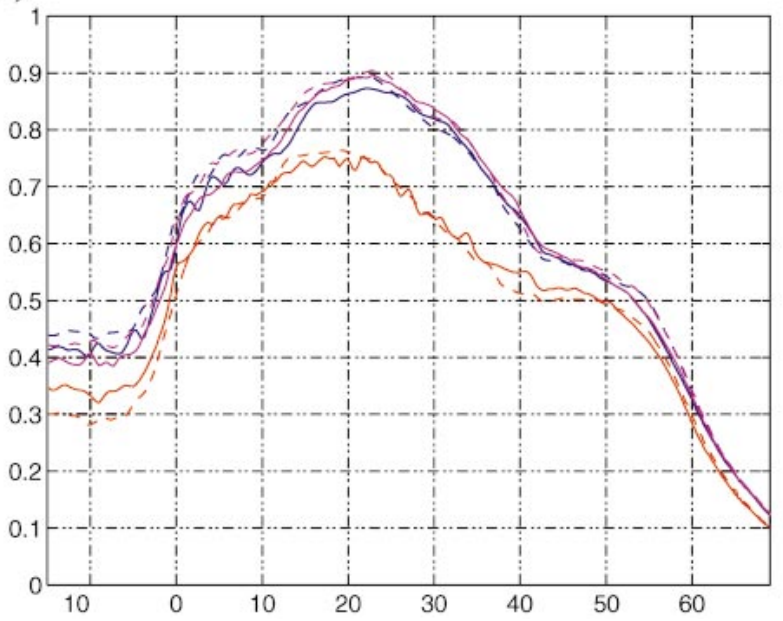

b)

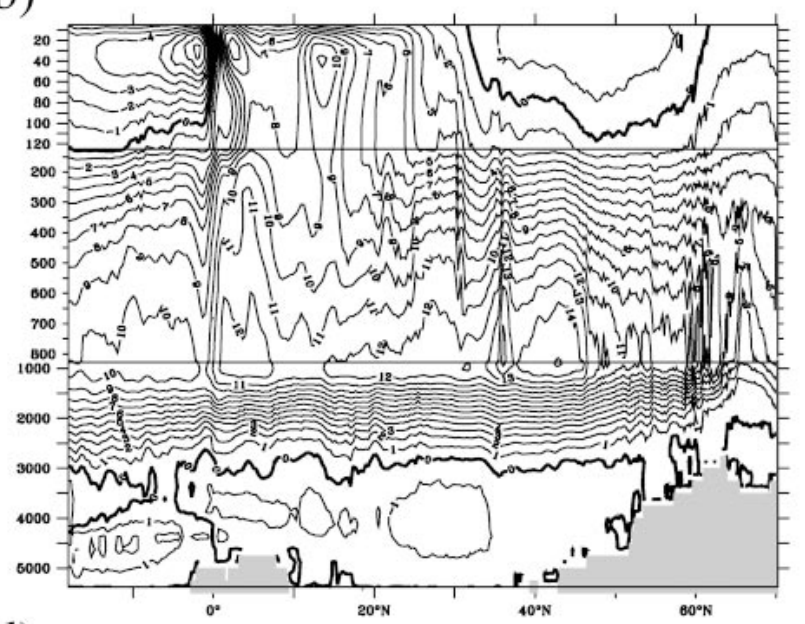

d)

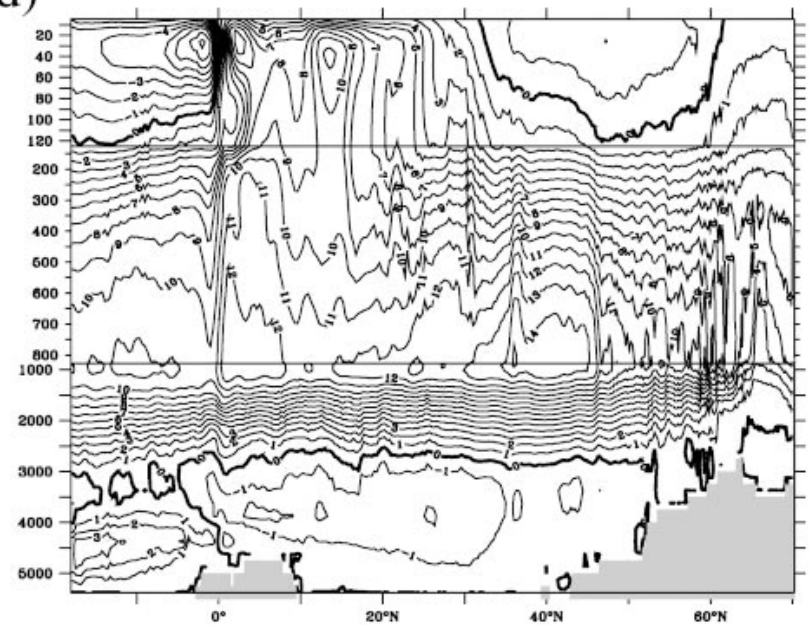

FIG. 10. (a) Mean northward heat transport in PW in the prognostic model (black), MEAN (red), SMOOTH (green), MEAN + SMOOTH (blue), and MEAN + SMOOTH-800 (magenta). (b) Meridional streamfunction in MEAN + SMOOTH-800 (Sv). Contour interval is $1 \mathrm{~Sv}$. (c) Mean northward heat transport in PW in MEAN (red, solid), MEAN-CORR (red, dashed), MEAN + SMOOTH (blue, solid), MEAN + SMOOTH-CORR (blue, dashed), MEAN + SMOOTH-800 (magenta, solid), and MEAN + SMOOTH-800-CORR (magenta, dashed). (d) Meridional streamfunction in MEAN + SMOOTH-800-CORR (Sv). Contour interval is $1 \mathrm{~Sv}$.

names in section $3 \mathrm{c}$ to identify these experiments; for example, the experiment utilizing the correction of MEAN is called MEAN-CORR.

First, we note that there are no large trends over the integration period of the experiments described in this section; that is, the solution of the modified semiprognostic models remains almost unchanged, switching to the corrected-prognostic version. For clarification, Fig. $10 \mathrm{c}$ shows again the heat transport in MEAN, MEAN + SMOOTH, and MEAN-SMOOTH-800 together with the corresponding corrected-prognostic experiments. We see that the heat transport in the corrected-prognostic versions remain at about the same level as in the corresponding semiprognostic version. The same holds for the other quantities discussed such as EKE, overturning, flow patterns, and so on.

Figures $9 \mathrm{~d}-\mathrm{f}$ show some examples of the solution of
MEAN + SMOOTH-800-CORR. Overall, we see that the benefits of the original semiprognostic model, as well as the modified versions, are carried over to the corrected-prognostic models. However, despite the small increase in northward heat transport in MEAN + SMOOTH-800-CORR, a little more heat gain shows up north of about $50^{\circ} \mathrm{N}$ in comparison with the corresponding modified semiprognostic version (cf. Fig. 9c), most likely due to a small southward shift of the NAC. However, there is still a large improvement in the pattern of the surface heat flux with respect to the prognostic model (cf. Fig. 3b) and, in addition, up to 0.1 PW more northward heat transport in the corrected-prognostic model, which is now well inside the error estimates of the midlatitude observations (cf. Fig. 6a). Note that with the corrected-prognostic model we finally resolve the problem of changed dynamical properties in the model 
(caveat 1). This effect is readily demonstrated in idealized numerical and analytical models, but we do not make any attempt to show the effect in our realistic OGCM.

\section{Concluding discussion}

We have applied the semiprognostic method as proposed by Sheng et al. (2001) to an eddy-permitting model of the North Atlantic. Our aim is to reduce systematic model errors, such as unrealistic upper-level flow and watermass characteristics of the NAC around Newfoundland and the Gulf Stream region between Cape Hatteras and the Grand Banks that are known to be longstanding deficiencies in North Atlantic models leading to spurious large-scale surface heat fluxes and to a bias of too-low northward heat transport (Böning et al. 1996; Willebrand et al. 2001). While model efforts with highly increased horizontal (eddy resolving) resolution show some improvements in this respect, results still crucially depend on model details such as parameterizations for friction and diffusion and discretization of topography (Smith et al. 2000; Chassignet and Garraffo 2001). We propose to focus research on this problem to understand and parameterize the underlying processes in the western boundary flow.

As for the regional model of Sheng et al. (2001), the semiprognostic model version performs better than the prognostic version with respect to observations at these key points of the North Atlantic circulation. In fact, the semiprognostic version is similar to an eddy-resolving model version in many respects. The improvement is achieved by applying a flow-interactive correction of the pressure gradient in the momentum budget, simply taken from hydrographic observations. Tracer budgets are not directly affected by the method; that is, no diabatic sources and sinks of heat or salt are introduced.

However, we have noted three inherent drawbacks of a semiprognostic model. These are changed dynamical properties of the model, that is, reduced geostrophic wave speeds and damped mesoscale eddy activity, and spurious interaction of the method with topography. We have discussed and successfully applied four simple modifications of the original semiprognostic method to overcome these drawbacks. By spatial averaging and/ or temporal averaging of the semiprognostic flow correction it is possible to include only the large-scale hydrographic information and to reduce the damping influence of the original method on eddy activity. In fact, eddy kinetic energy is even enhanced in the modified semiprognostic models (more than $30 \%$ for the mean method) in comparison with the prognostic model. In combination with a relaxation of the flow correction near the coast, it is possible to overcome the spurious interaction with the topography. Restricting the flow correction furthermore to the upper ocean (roughly in the permanent thermocline), thus excluding the (even- tually) less reliable ${ }^{9}$ deep observations, reveals essentially the same benefits as using the flow correction everywhere.

Having done a spinup integration with one or a combination of the modified methods in a semiprognostic model, we proceed by diagnosing the correction term and applying it in the subsequent integration (correctedprognostic model), resolving the problem of modified physical properties and acting similar to a parameterization of unresolved processes in the OGCM. The benefits of the original semiprognostic model, that is, a better representation of the advective flow east of Newfoundland and in the Gulf Stream region, are carried over to (or are even outperformed by) the modified semiprognostic models and, finally, to our end product, the corrected-prognostic model. We also note that similar benefits of the method can be obtained in non-eddyresolving OGCMs, as experiments with a version of our OGCM with coarser resolution $\left(4 / 3^{\circ} \cos \phi\right)$ reveal; however, this result is not discussed in the present study.

Motivation for correcting the OGCM is the largescale spurious surface heat fluxes (cf. Fig. 3) of models of the North Atlantic, recognized as a long-standing deficiency in many previous model studies (Böning et al. 1996; Willebrand et al. 2001), along with a bias of too-low northward heat transport. In particular, spurious heat fluxes are related to a missing northward turn of the NAC as it flows southeast of Newfoundland, showing up in eddy-permitting OGCMs (but also, to a sometimes even greater extent, in non-eddy-resolving models). We argue that using such an eddy-permitting (uncorrected) OGCM in a coupled model system might not improve the realism of the simulation in comparison with the use of a non-eddy-resolving OGCM, at least in this ("storm track formation") region, which is of potential importance for air-sea interaction. Unless a suitable parameterization is found, the correction strategy for the OGCM presented in this study might allow one to simulate the correct position of the subpolar front in the western North Atlantic, leading to a more realistic coupled simulation of the atmospheric storm track in this region.

To quantify the effect, we calculate the horizontal average over the region east of Newfoundland $\left(42^{\circ}-\right.$ $\left.50^{\circ} \mathrm{N}, 42^{\circ}-30^{\circ} \mathrm{W}\right)$, in which the NAC loses large amounts of heat to the atmosphere, $57 \mathrm{TW}$ according to the heat fluxes of the ECMWF analysis (Barnier et al. 1995). In contrast, the prognostic model gains 19 TW in this region because of the missing northwest turn of the NAC, similar to, for example, the DYNAMO zlevel model of Willebrand et al. (2001) and many other previous model solutions. The modified semiprognostic model (MEAN + SMOOTH-800) reduces this systematic error of $76 \mathrm{TW}$ through adiabatically changing the

\footnotetext{
${ }^{9}$ We mean by "less reliable" a potentially larger error at depth due a smaller number of observations that are insufficient to filter out effects of eddy variability.
} 
advective flow in this region by about $43 \%$, and the corresponding corrected-prognostic model by $25 \%$, both leading now to an average heat loss of the model in this region. Furthermore, the total northward heat transport increases in the corrected-prognostic model by about $0.1 \mathrm{PW}$ (and to a similar extent in the modified semiprognostic model) in comparison with the uncorrected prognostic model.

We must note, however, that, since we are using a fixed non-flow-interactive correction of the large-scale properties of the model based on historic hydrographic observations, the corrected-prognostic model is not well suited for coupled simulations with large deviations from the present climate. However, it should be well suited for simulations of the present climate state, for example, seasonal- to decadal-scale climate forecasts or hindcasts. In a simulation of, for instance, the last glacial maximum or serious climate changes due to greenhouse gas forcing, in which large deviations of the mean, largescale oceanic flow can be expected, one has to fall back to the prognostic OGCM. On the other hand, flux correcting the OGCM in such a coupled simulation contains information about the present climate state in a very similar way as our approach, thus suffering from the same problem.

However, in addition to a coupled climate model, another attractive possible application of the method is the following. Since the correction in a semiprognostic, or corrected-prognostic, model does not affect the tracer budgets directly, it would also be well suited for the simulation of the advective, turbulent transport of a passive tracer in a realistic ocean model of the present climate state. Possible applications are for example the simulation of the uptake of anthropogenic $\mathrm{CO}_{2}$, coupling of the OGCM to pelagic ecosystem models, or oceanic now- and forecasts for industrial purposes, for example, a simulation of the dispersal of pollutants.

Last, we note that the semiprognostic method is well suited to be used in a pragmatic, two-way nesting approach. In particular, the transfer of information from a nested model with enhanced resolution to a model of a larger domain with coarser resolution is usually difficult to realize. However, constraining the nested model to match the coarser-resolution model at its boundaries can also cause problems; that is, the nested model can get "decoupled" from its boundary conditions. Clearly, the semiprognostic method is a simple and robust way to adiabatically pass large-scale information from the larger domain into the nested model and small-scale information of the nested model to the larger domain. We will explore this route in a future study.

Acknowledgments. This work has been supported by funding provided to the Canadian CLIVAR Research Network by NSERC, CFCAS, and CICS. RJG is also supported by the NSERC/MARTEC/MSC Industrial Research Chair. We are grateful for the use of computer facilities at the Canadian Meteorological Centre in Dor- val, Quebec, Canada, and we acknowledge the contributions of the FLAME group at IfM Kiel. The integration of the eddy-resolving model was performed at the German high-performance computing centers ZIB Berlin and LRZ Munich, supported by the German CLIVAR program. Comments from two anonymous reviewers and the editor were helpful for improving the manuscript.

\section{REFERENCES}

Barnier, B., L. Siefridt, and P. Marchesiello, 1995: Thermal forcing for a global ocean circulation model using a three year climatology of ECMWF analysis. J. Mar. Syst., 6, 363-380.

Böning, C. W., W. R. Holland, F. O. Bryan, G. Danabasoglu, and J. C. McWilliams, 1995: An overlooked problem in model simulations of the thermohaline circulation and heat transport in the Atlantic Ocean. J. Climate, 8, 515-523.

_ , F. O. Bryan, W. R. Holland, and R. Doescher, 1996: Deepwater formation and meridional overturning in a high-resolution model of the North Atlantic. J. Phys. Oceanogr., 26, 1142-1164.

Boyer, T. P., and S. Levitus, 1997: Objective analyses of temperature and salinity for the World Ocean on a 1/4 degree grid. NOAA Atlas NESDIS 11, CD-ROM.

Chassignet, E. P., and Z. D. Garraffo, 2001: Viscosity parameterization and the Gulf Stream separation. From Stirring to Mixing in a Stratified Ocean: Proc. 'Aha Huliko'a Hawaiian Winter Workshop, Honolulu, HI, University of Hawaii at Manoa, 3741.

Dengg, J., A. Beckmann, and R. Gerdes, 1996: The Gulf Stream separation problem. The Warmwatersphere of the North Atlantic Ocean, W. Krauss, Ed., Gebrueder Borntraeger, 253-290.

— C. W. Böning, U. Ernst, R. Redler, and A. Beckmann, 1999: Effects of an improved model representation of overflow water on the subpolar North Atlantic. International WOCE Newsletter. No. 37, WOCE International Project Office, Southampton, United Kingdom, 10-15.

Eden, C., and C. W. Böning, 2002: Sources of eddy kinetic energy in the Labrador Sea. J. Phys. Oceanogr., 32, 3346-3363.

_ and R. J. Greatbatch, 2003: A damped decadal oscillation in the North Atlantic climate system. J. Climate, 16, 4043-4068.

Ezer, T., and G. L. Mellor, 1994: Diagnostic and prognostic calculations of the North Atlantic circulation and sea level using a sigma coordinate ocean model. J. Geophys. Res., 99 (C7), 14 159-14 171.

FLAME Group, 1998: FLAME-A Family of Linked Atlantic Model Experiments. AWI Tech. Rep., Bremerhaven, Germany, 89 pp. [Available online at ftp://ftp.ifm.uni-kiel.de/pub/FLAME/ WebDownload/Reports/FLAME_Rep 98.ps.gz.]

Ganachaud, A., and C. Wunsch, 2000: Improved estimates of global ocean circulation, heat transport and mixing from hydrographic data. Nature, 408, 453-457.

Gargett, A. E., 1984: Vertical eddy diffusivity in the ocean interior. J. Mar. Res., 42, 359-393.

Gaspar, P., Y. Gregoris, and J.-M. Lefevre, 1990: A simple eddy kinetic energy model for simulations of the oceanic vertical mixing: Tests at station PAPA and Long-Term Upper Ocean Study site. J. Geophys. Res., 95, 16 179-16 193.

Geshelin, Y., J. Sheng, and R. J. Greatbatch, 1999: Monthly mean climatologies of temperature and salinity in the western North Atlantic. Canadian Data Report of Hydrography and Ocean Sciences No. 153.

Greatbatch, R. J., A. F. Fanning, A. D. Goulding, and S. Levitus, 1991: A diagnosis of interpentadal circulation changes in the North Atlantic. J. Geophys. Res., 96, 22 009-22 023.

Griffies, S. M., 1998: The Gent-McWilliams skew flux. J. Phys. Oceanogr., 28, 831-841.

, R. C. Pacanowski, and B. R. Hallberg, 2000: Spurious diapycnal 
mixing associated with advection in a $z$-coordinate ocean model. Mon. Wea. Rev., 128, 538-564.

Hoskins, B. J., and P. J. Valdes, 1990: On the existence of storm tracks. J. Atmos. Sci., 47, 1854-1864.

Kröger, J., 2001: Mechanismen meridionaler Transportprozesse im tropischen Atlantik. Ph.D. thesis, Institut für Meereskunde, Universität Kiel, 153 pp.

Levitus, S., and T. P. Boyer, 1994: Temperature. Vol. 4, World Ocean Atlas 1994, NOAA Atlas NESDIS 4, $117 \mathrm{pp}$

MacDonald, A. M., and C. Wunsch, 1996: An estimate of global ocean circulation and heat fluxes. Nature, 382, 436-439.

Marotzke, J., and J. Willebrand, 1996: The North Atlantic mean circulation: Combining data and dynamics. The Warmwatersphere of the North Atlantic Ocean, W. Krauss, Ed., Gebrueder Borntraeger, 55-90.

Oschlies, A., and V. Garcon, 1999: An eddy-permitting coupled physical-biological model of the North Atlantic. 1. Sensitivity to advection numerics and mixed layer physics. Global Biogeochem. Cycles, 13, 135-160.

Pacanowski, R. C., 1995: MOM 2 documentation, user's guide and reference manual. GFDL Ocean Group Tech. Rep., GFDL, Princeton, NJ, 329 pp.

Paiva, A. M., J. T. Hargrove, E. P. Chassignet, and R. Bleck, 1999 Turbulent behavior of a fine mesh (1/12 degree) numerical simulation of the North Atlantic. J. Mar. Syst., 21, 307-320.

Sarmiento, J. L., and K. Bryan, 1982: An ocean transport model for the North Atlantic. J. Geophys. Res., 87, 394-408.
Sheng, J., R. J. Greatbatch, and D. Wright, 2001: Improving the utility of ocean circulation models through adjustment of the momentum balance. J. Geophys. Res., 106, 16 711-16 728.

Smith, R. D., M. E. Maltrud, F. O. Bryan, and M. W. Hecht, 2000 Numerical simulation of the North Atlantic Ocean at $1 / 10^{\circ} . J$. Phys. Oceanogr., 30, 1532-1561.

Stammer, D., P. R. Tokmakian, A. Semnter, and C. Wunsch, 1996: How well does a $1 / 4^{\circ}$ global circulation model simulate large scale oceanic observations? J. Geophys. Res., 101, 25 779-25 811.

Stevens, D. P., 1990: On open boundary conditions for three dimensional primitive equation ocean circulation models. Geophys. Astrophys. Fluid Dyn., 51, 103-133.

Stutzer, S., and W. Krauss, 1998: Mean circulation and transports in the South Atlantic Ocean: Combining model and drifter data. $J$. Geophys. Res., 103, 30 985-31002.

Trenberth, K., and J. Caron, 2001: Estimates of meridional atmosphere and ocean heat transports. J. Climate, 14, 3433-3443.

Veronis, G., 1975: The role of models in tracer studies. Numerical Models of the Ocean Circulation, National Academy of Sciences, 133-146.

Willebrand, J., and Coauthors, 2001: Circulation characteristics in three eddy-permitting models of the North Atlantic. Progress in Oceanography, Vol. 48, Pergamon, 123-161.

Woodgate, R. A., and P. D. Killworth, 1997: The effects of assimilation on the physics of an ocean model. Part I: Theoretical model and barotropic results. J. Atmos. Oceanic Technol., 14, 897-909. 